\title{
Fortran programs for the time-dependent Gross-Pitaevskii equation in a fully anisotropic trap
}

\author{
P. Muruganandam ${ }^{\mathrm{a}, \mathrm{b}}$ and S. K. Adhikari ${ }^{\mathrm{b}, *}$ \\ ${ }^{a}$ School of Physics, Bharathidasan University, Palkalaiperur Campus, Tiruchirappalli - 620024, Tamil Nadu, India \\ ${ }^{\mathrm{b}}$ Instituto de Física Teórica, UNESP - São Paulo State University, Barra Funda, 01.140-70 São Paulo, São Paulo, Brazil
}

\begin{abstract}
Here we develop simple numerical algorithms for both stationary and non-stationary solutions of the timedependent Gross-Pitaevskii (GP) equation describing the properties of Bose-Einstein condensates at ultra low temperatures. In particular, we consider algorithms involving real- and imaginary-time propagation based on a split-step Crank-Nicolson method. In a one-space-variable form of the GP equation we consider the one-dimensional, twodimensional circularly-symmetric, and the three-dimensional spherically-symmetric harmonic-oscillator traps. In the two-space-variable form we consider the GP equation in two-dimensional anisotropic and three-dimensional axiallysymmetric traps. The fully-anisotropic three-dimensional GP equation is also considered. Numerical results for the chemical potential and root-mean-square size of stationary states are reported using imaginary-time propagation programs for all the cases and compared with previously obtained results. Also presented are numerical results of non-stationary oscillation for different trap symmetries using real-time propagation programs. A set of convenient working codes developed in Fortran 77 are also provided for all these cases (twelve programs in all). In the case of two or three space variables, Fortran 90/95 versions provide some simplification over the Fortran 77 programs, and these programs are also included (six programs in all).
\end{abstract}

Key words: Bose-Einstein condensate; Gross-Pitaevskii equation; Split-step Crank-Nicolson Scheme; Real- and imaginary-time propagation; Fortran program; Partial differential equation

PACS: 02.60.Lj; 02.60.Jh; 02.60.Cb; 03.75.-b

\section{Program summary (1)}

Title of program: imagtime1d.F

Title of electronic file: imagtime1d.tar.gz

Catalogue identifier:

Program summary URL:

Program obtainable from: CPC Program Library, Queen's University of Belfast, N. Ireland

Distribution format: tar.gz

Computers: PC/Linux, workstation/UNIX

Maximum Ram Memory: 1 GByte

Programming language used: Fortran 77

\footnotetext{
* Corresponding author; address: Instituto de Física Teórica, Rua Pamplona 145, 01405-900 São Paulo, SP, Brazil; telephone: $+551131779071$

Email addresses: anand@cnld.bdu.ac.in (P. Muruganandam), adhikari@ift.unesp.br (S. K. Adhikari).
} 
Typical running time: Minutes on a medium PC

Unusual features: None

Nature of physical problem: This program is designed to solve the time-dependent Gross-Pitaevskii nonlinear partial differential equation in one space dimension with a harmonic trap. The Gross-Pitaevskii equation describes the properties of a dilute trapped Bose-Einstein condensate.

Method of solution: The time-dependent Gross-Pitaevskii equation is solved by the split-step CrankNicolson method by discretizing in space and time. The discretized equation is then solved by propagation in imaginary time over small time steps. The method yields the solution of stationary problems.

Program summary (2)

Title of program: imagtimecir.F

Title of electronic file: imagtimecir.tar.gz

Catalogue identifier:

Program summary URL:

Program obtainable from: CPC Program Library, Queen's University of Belfast, N. Ireland

Distribution format: tar.gz

Computers: PC/Linux, workstation/UNIX

Maximum Ram Memory: 1 GByte

Programming language used: Fortran 77

Typical running time: Minutes on a medium PC

Unusual features: None

Nature of physical problem: This program is designed to solve the time-dependent Gross-Pitaevskii nonlinear partial differential equation in two space dimensions with a circularly-symmetric trap. The GrossPitaevskii equation describes the properties of a dilute trapped Bose-Einstein condensate.

Method of solution: The time-dependent Gross-Pitaevskii equation is solved by the split-step CrankNicolson method by discretizing in space and time. The discretized equation is then solved by propagation in imaginary time over small time steps. The method yields the solution of stationary problems.

Program summary (3)

Title of program: imagtimesph.F

Title of electronic file: imagtimesph.tar.gz

Catalogue identifier:

Program summary URL:

Program obtainable from: CPC Program Library, Queen's University of Belfast, N. Ireland

Distribution format: tar.gz

Computers: PC/Linux, workstation/UNIX

Maximum Ram Memory: 1 GByte

Programming language used: Fortran 77

Typical running time: Minutes on a medium PC

Unusual features: None

Nature of physical problem: This program is designed to solve the time-dependent Gross-Pitaevskii nonlinear partial differential equation in three space dimensions with a spherically-symmetric trap. The GrossPitaevskii equation describes the properties of a dilute trapped Bose-Einstein condensate.

Method of solution: The time-dependent Gross-Pitaevskii equation is solved by the split-step CrankNicolson method by discretizing in space and time. The discretized equation is then solved by propagation in imaginary time over small time steps. The method yields the solution of stationary problems.

Program summary (4)

Title of program: realtime1d.F

Title of electronic file: realtime1d.tar.gz

Catalogue identifier:

Program summary URL:

Program obtainable from: CPC Program Library, Queen's University of Belfast, N. Ireland

Distribution format: tar.gz

Computers: PC/Linux, workstation/UNIX 
Maximum Ram Memory: 2 GByte

Programming language used: Fortran 77

Typical running time: Minutes on a medium PC

Unusual features: None

Nature of physical problem: This program is designed to solve the time-dependent Gross-Pitaevskii nonlinear partial differential equation in one space dimension with a harmonic trap. The Gross-Pitaevskii equation describes the properties of a dilute trapped Bose-Einstein condensate.

Method of solution: The time-dependent Gross-Pitaevskii equation is solved by the split-step CrankNicolson method by discretizing in space and time. The discretized equation is then solved by propagation in real time over small time steps. The method yields the solution of stationary and non-stationary problems.

Program summary (5)

Title of program: realtimecir.F

Title of electronic file: realtimecir.tar.gz

Catalogue identifier:

Program summary URL:

Program obtainable from: CPC Program Library, Queen's University of Belfast, N. Ireland

Distribution format: tar.gz

Computers: PC/Linux, workstation/UNIX

Maximum Ram Memory: 2 GByte

Programming language used: Fortran 77

Typical running time: Minutes on a medium PC

Unusual features: None

Nature of physical problem: This program is designed to solve the time-dependent Gross-Pitaevskii nonlinear partial differential equation in two space dimensions with a circularly-symmetric trap. The GrossPitaevskii equation describes the properties of a dilute trapped Bose-Einstein condensate.

Method of solution: The time-dependent Gross-Pitaevskii equation is solved by the split-step CrankNicolson method by discretizing in space and time. The discretized equation is then solved by propagation in real time over small time steps. The method yields the solution of stationary and non-stationary problems.

Program summary (6)

Title of program: realtimesph.F

Title of electronic file: realtimesph.tar.gz

Catalogue identifier:

Program summary URL:

Program obtainable from: CPC Program Library, Queen's University of Belfast, N. Ireland

Distribution format: tar.gz

Computers: PC/Linux, workstation/UNIX

Maximum Ram Memory: 2 GByte

Programming language used: Fortran 77

Typical running time: Minutes on a medium PC

Unusual features: None

Nature of physical problem: This program is designed to solve the time-dependent Gross-Pitaevskii nonlinear partial differential equation in three space dimensions with a spherically-symmetric trap. The GrossPitaevskii equation describes the properties of a dilute trapped Bose-Einstein condensate.

Method of solution: The time-dependent Gross-Pitaevskii equation is solved by the split-step CrankNicolson method by discretizing in space and time. The discretized equation is then solved by propagation in real time over small time steps. The method yields the solution of stationary and non-stationary problems.

Program summary (7)

Title of programs: imagtimeaxial.F and imagtimeaxial.f90

Title of electronic file: imagtimeaxial.tar.gz

Catalogue identifier:

Program summary URL:

Program obtainable from: CPC Program Library, Queen's University of Belfast, N. Ireland 
Distribution format: tar.gz

Computers: PC/Linux, workstation/UNIX

Maximum Ram Memory: 2 GByte

Programming language used: Fortran 77 and Fortran 90

Typical running time: Few hours on a medium PC

Unusual features: None

Nature of physical problem: This program is designed to solve the time-dependent Gross-Pitaevskii nonlinear partial differential equation in three space dimensions with an axially-symmetric trap. The GrossPitaevskii equation describes the properties of a dilute trapped Bose-Einstein condensate.

Method of solution: The time-dependent Gross-Pitaevskii equation is solved by the split-step CrankNicolson method by discretizing in space and time. The discretized equation is then solved by propagation in imaginary time over small time steps. The method yields the solution of stationary problems.

Program summary (8)

Title of program: imagtime2d.F and imagtime2d.f90

Title of electronic file: imagtime2d.tar.gz

Catalogue identifier:

Program summary URL:

Program obtainable from: CPC Program Library, Queen's University of Belfast, N. Ireland

Distribution format: tar.gz

Computers: PC/Linux, workstation/UNIX

Maximum Ram Memory: 2 GByte

Programming language used: Fortran 77 and Fortran 90

Typical running time: Few hours on a medium PC

Unusual features: None

Nature of physical problem: This program is designed to solve the time-dependent Gross-Pitaevskii nonlinear partial differential equation in two space dimensions with an anisotropic trap. The Gross-Pitaevskii equation describes the properties of a dilute trapped Bose-Einstein condensate.

Method of solution: The time-dependent Gross-Pitaevskii equation is solved by the split-step CrankNicolson method by discretizing in space and time. The discretized equation is then solved by propagation in imaginary time over small time steps. The method yields the solution of stationary problems.

Program summary (9)

Title of program: realtimeaxial.F and realtimeaxial.f90

Title of electronic file: realtimeaxial.tar.gz

Catalogue identifier:

Program summary URL:

Program obtainable from: CPC Program Library, Queen's University of Belfast, N. Ireland

Distribution format: tar.gz

Computers: PC/Linux, workstation/UNIX

Maximum Ram Memory: 4 GByte

Programming language used: Fortran 77 and Fortran 90

Typical running time: Hours on a medium PC

Unusual features: None

Nature of physical problem: This program is designed to solve the time-dependent Gross-Pitaevskii nonlinear partial differential equation in three space dimensions with an axially-symmetric trap. The GrossPitaevskii equation describes the properties of a dilute trapped Bose-Einstein condensate.

Method of solution: The time-dependent Gross-Pitaevskii equation is solved by the split-step CrankNicolson method by discretizing in space and time. The discretized equation is then solved by propagation in real time over small time steps. The method yields the solution of stationary and non-stationary problems.

Program summary (10)

Title of program: realtime2d.F and realtime2d.f90

Title of electronic file: realtime2d.tar.gz

Catalogue identifier: 
Program summary URL:

Program obtainable from: CPC Program Library, Queen's University of Belfast, N. Ireland

Distribution format: tar.gz

Computers: PC/Linux, workstation/UNIX

Maximum Ram Memory: 4 GByte

Programming language used: Fortran 77 and Fortran 90

Typical running time: Hours on a medium PC

Unusual features: None

Nature of physical problem: This program is designed to solve the time-dependent Gross-Pitaevskii nonlinear partial differential equation in two space dimensions with an anisotropic trap. The Gross-Pitaevskii equation describes the properties of a dilute trapped Bose-Einstein condensate.

Method of solution: The time-dependent Gross-Pitaevskii equation is solved by the split-step CrankNicolson method by discretizing in space and time. The discretized equation is then solved by propagation in real time over small time steps. The method yields the solution of stationary and non-stationary problems.

Program summary (11)

Title of program: imagtime3d.F and imagtime3d.f90

Title of electronic file: imagtime3d.tar.gz

Catalogue identifier:

Program summary URL:

Program obtainable from: CPC Program Library, Queen's University of Belfast, N. Ireland

Distribution format: tar.gz

Computers: PC/Linux, workstation/UNIX

Maximum Ram Memory: 4 GByte

Programming language used: Fortran 77 and Fortran 90

Typical running time: Few days on a medium PC

Unusual features: None

Nature of physical problem: This program is designed to solve the time-dependent Gross-Pitaevskii nonlinear partial differential equation in three space dimensions with an anisotropic trap. The Gross-Pitaevskii equation describes the properties of a dilute trapped Bose-Einstein condensate.

Method of solution: The time-dependent Gross-Pitaevskii equation is solved by the split-step CrankNicolson method by discretizing in space and time. The discretized equation is then solved by propagation in imaginary time over small time steps. The method yields the solution of stationary problems.

Program summary (12)

Title of program: realtime3d.F and realtime 3 d.f90

Title of electronic file: realtime3d.tar.gz

Catalogue identifier:

Program summary URL:

Program obtainable from: CPC Program Library, Queen's University of Belfast, N. Ireland

Distribution format: tar.gz

Computers: PC/Linux, workstation/UNIX

Maximum Ram Memory: 8 GByte

Programming language used: Fortran 77 and Fortran 90

Typical running time: Days on a medium PC

Unusual features: None

Nature of physical problem: This program is designed to solve the time-dependent Gross-Pitaevskii nonlinear partial differential equation in three space dimensions with an anisotropic trap. The Gross-Pitaevskii equation describes the properties of a dilute trapped Bose-Einstein condensate.

Method of solution: The time-dependent Gross-Pitaevskii equation is solved by the split-step CrankNicolson method by discretizing in space and time. The discretized equation is then solved by propagation in real time over small time steps. The method yields the solution of stationary and non-stationary problems. 


\section{Introduction}

After a successful experimental detection of Bose-Einstein condensates (BEC) of dilute trapped bosonic alkali-metal atoms ${ }^{7} \mathrm{Li},{ }^{23} \mathrm{Na}$, and ${ }^{87} \mathrm{Rb}[1,2]$ at ultra-low temperatures, there have been intense theoretical activities in studying properties of the condensate using the time-dependent mean-field Gross-Pitaevskii (GP) equation under different trap symmetries. Among many possibilities, the following traps have been used in various studies: three-dimensional (3D) spherically-symmetric, axially-symmetric and anisotropic harmonic traps, two-dimensional (2D) circularly-symmetric and anisotropic harmonic traps, and one-dimensional (1D) harmonic trap. The inter-atomic interaction leads to a nonlinear term in the GP equation, which complicates its accurate numerical solution, specially for a large nonlinearity. The nonlinearity is large for a fixed harmonic trap when either the number of atoms in the condensate or the atomic scattering length is large and this is indeed so under many experimental conditions. Special care is needed for the solution of the time-dependent GP equation with large nonlinearity and there has been an extensive literature on this topic [3-48].

The time-dependent GP equation is a partial differential equation in space and time variables involving first-order time and second-order space derivatives together with a harmonic and a nonlinear potential term, and has the structure of a nonlinear Schrödinger equation with a harmonic trap. One commonly used procedure for the solution of the time-dependent GP equation makes use of a discretization of this equation in space and time and subsequent integration and time propagation of the discretized equation. From a knowledge of the solution of this equation at a specific time, this procedure finds the solution after a small time step by solving the discretized equation. A commonly used discretization scheme for the GP equation is the semi-implicit Crank-Nicolson discretization scheme [49-51] which has certain advantages and will be used in this work.

In the simplest one-space-variable form of the GP equation, the solution algorithm is executed in two steps. In the first step, using a known initial solution, an intermediate solution after a small interval of time $\Delta$ is found neglecting the harmonic and nonlinear potential terms. The effect of the potential terms is then included by a first-order time integration to obtain the final solution after time $\Delta$. In case of two or three spatial variables, the space derivatives are dealt with in two or three steps and the effect of the potential terms are included next. As the time evolution is executed in different steps it is called a split-step real-time propagation method. This method is equally applicable to stationary ground and excited states as well as non-stationary states, although in this paper we do not consider stationary excited states. The virtues of the semi-implicit Crank-Nicolson scheme [49-51] are that it is unconditionally stable and preserves the normalization of the solution under real-time propagation. A simpler and efficient variant of the scheme called the split-step imaginary-time propagation method obtained by replacing the time variable by an imaginary time is also considered. (The GP equation involves complex variables. However, after replacing the time variable by an imaginary time the resultant partial differential equation is real, and hence the imaginarytime propagation method involves real variables only. This trick leads to an imaginary-time operator which results in exponential decay of all states relative to the ground state and can then be applied to any initial trial wave function to compute an approximation to the actual ground state rather accurately. We shall use imaginary-time propagation to compute the ground state in this paper.) The split-step imaginary-time propagation method involving real variables yields very precise result at low computational cost (CPU time) and is very appropriate for the solution of stationary problems involving the ground state. The split-step real-time propagation method uses complex quantities and yields less precise results for stationary problems; however, they are appropriate for the study of non-equilibrium dynamics in addition to stationary problems involving excited states also.

Most of the previous studies [3-5,8,9,12,18,20,21,24,25,33-35,39,45,46] on the numerical solution of the GP equation are confined to a consideration of stationary states only. Some used specifically the imaginary-time propagation method $[6,31,44,45]$. There are few studies $[30,37,38,41]$ for the numerical solution of the timedependent GP equation using the Crank-Nicolson method [49-51]. Other methods for numerical solution of the time-dependent GP equation have also appeared in the literature $[7,14,16,23,26-29,32,42,43,47,48]$. These time-dependent methods can be used for studying non-equilibrium dynamics of the condensate involving 
non-stationary states.

The purpose of the present paper is to develop a simple and efficient algorithm for the numerical solution of the GP equation using time propagation together with the semi-implicit Crank-Nicolson discretization scheme [49-51] specially useful to newcomers in this field interested in obtaining a numerical solution of the time-dependent GP equation. Easy-to-use Fortran 77 programs for different trap symmetries with adequate explanation are also included. In case of two and three space variables, Fortran 90/95 programs are more compact in nature and these programs are also included. We include programs using both real- and imaginary-time propagation. For stationary ground states the imaginary-time method has a much quicker convergence rate compared to the real-time method and should be used for the calculation of chemical potential, energy and root-mean-square ( $\mathrm{rms}$ ) sizes. We calculate the chemical potential and rms sizes of the condensate for stationary problems and compare these results with those previously obtained by other workers for different trap symmetries. These results can be easily calculated in a decent PC using the Fortran programs provided. In addition to the results for the stationary states, the real-time propagation routines can also be used to study the non-stationary transitions, as in collapse dynamics [52] and non-equilibrium oscillation [30].

This paper is organized as follows. In Sec. 2 we present the GP equations with different traps that we consider in this paper, e.g., the 3D spherically-symmetric, 2D circularly-symmetric and 1D harmonic traps involving one space variable, the anisotropic 2D and axially-symmetric 3D harmonic traps in two space variables and the fully anisotropic $3 \mathrm{D}$ harmonic trap in three space variables. In Sec. 3 we elaborate the numerical algorithm for solving the GP equation in one space variable (the 1D, circularly-symmetric 2D, and spherically-symmetric 3D cases) and for calculating the chemical potential, energy and rms sizes employing both the real- and imaginary-time propagation methods. In Sec. 4 we present the same for solving the GP equation in two and three space variables (the anisotropic $2 \mathrm{D}$ and axially-symmetric and anisotropic $3 \mathrm{D}$ cases). In Sec. 5 we present a description of the Fortran programs, an explanation about how to use them, and some sample outputs. In Sec. 6 we present the numerical results for chemical potential, rms size, value of the wave function at the center, for the ground-state problem using the imaginary-time propagation routines and compare our finding with previous results for different trap symmetries in 1D, 2D, and 3D. We also present a study of non-stationary oscillation in some of these cases using the real-time propagation routines when the nonlinear coefficient in the GP equation with a stationary solution was suddenly reduced to half its value. Finally, in Sec. 7 we present a brief summary of our study.

\section{Nonlinear Gross-Pitaevskii Equation}

At zero temperature, the time-dependent Bose-Einstein condensate wave function $\Psi \equiv \Psi(\mathbf{r} ; \tau)$ at position $\mathbf{r}$ and time $\tau$ may be described by the following mean-field nonlinear GP equation [1]

$$
\mathrm{i} \hbar \frac{\partial \Psi(\mathbf{r} ; \tau)}{\partial \tau}=\left[-\frac{\hbar^{2} \nabla^{2}}{2 m}+V(\mathbf{r})+g N|\Psi(\mathbf{r} ; \tau)|^{2}\right] \Psi(\mathbf{r} ; \tau)
$$

with $\mathrm{i}=\sqrt{-1}$. Here $m$ is the mass of an atom and $N$ the number of atoms in the condensate, $g=4 \pi \hbar^{2} a / m$ the strength of inter-atomic interaction, with $a$ the atomic scattering length. The normalization condition of the wave function is $\int d \mathbf{r}|\Psi(\mathbf{r} ; \tau)|^{2}=1$.

\subsection{Spherically-symmetric GP equation in $3 D$}

In this case the trap potential is given by $V(\mathbf{r})=\frac{1}{2} m \omega^{2} \tilde{r}^{2}$, where $\omega$ is the angular frequency and $\tilde{r}$ the radial distance. After a partial-wave projection the radial part $\psi$ of the wave function $\Psi$ can be written as $\Psi(\mathbf{r} ; \tau)=\psi(\tilde{r}, \tau)$. After a transformation of variables to dimensionless quantities defined by $r=\sqrt{2} \tilde{r} / l$, $t=\tau \omega, l \equiv \sqrt{(\hbar / m \omega)}$ and $\phi(r ; t) \equiv \varphi(r ; t) / r=\psi(\tilde{r}, \tau)\left[l^{3} /(2 \sqrt{2})\right]^{1 / 2}$, the GP equation (1) in this case becomes 


$$
\left[-\frac{\partial^{2}}{\partial r^{2}}+\frac{r^{2}}{4}+\aleph\left|\frac{\varphi(r ; t)}{r}\right|^{2}-\mathrm{i} \frac{\partial}{\partial t}\right] \varphi(r ; t)=0,
$$

where $\aleph=8 \sqrt{2} \pi N a / l$. The purpose of changing the wave function from $\psi$ to $\varphi=r \psi$ is a matter of taste and it has certain advantages. First, this transformation removes the first derivative $\partial / \partial r$ from the differential equation (2) and thus results in a simpler equation [34]. Secondly, at the origin $r=0, \psi$ is a constant, or $\partial \psi / \partial r=0$. But the new variable satisfies $\varphi(0, t)=0$. Hence, while solving the differential equation (2), we can implement the simple boundary condition that as $r \rightarrow 0$ or $\infty, \varphi$ vanishes. The boundary condition for the differential equation in $\psi$ will be a mixed one, e.g., the function $\psi$ should vanish at infinity and its first space derivative should vanish at the origin. The normalization condition for the wave function is

$$
4 \pi \int_{0}^{\infty} d r|\varphi(r ; t)|^{2}=1
$$

However, Eq. (2) is not the unique form of dimensionless GP equation in this case. Other forms of dimensionless equations have been obtained and used by different workers. For example, using the transformations $r=\tilde{r} / l, t=\tau \omega, l \equiv \sqrt{(\hbar / m \omega)}$ and $\phi(r ; t) \equiv \varphi(r ; t) / r=\psi(\tilde{r}, \tau) l^{3 / 2}$, the GP equation (1) becomes

$$
\left[-\frac{1}{2} \frac{\partial^{2}}{\partial r^{2}}+\frac{1}{2} r^{2}+\aleph\left|\frac{\varphi(r ; t)}{r}\right|^{2}-\mathrm{i} \frac{\partial}{\partial t}\right] \varphi(r ; t)=0,
$$

where $\aleph=4 \pi N a / l$ with normalization (3). Finally, using the transformations $r=\tilde{r} / l, t=\tau \omega / 2, l \equiv$ $\sqrt{(\hbar / m \omega)}$ and $\phi(r ; t) \equiv \varphi(r ; t) / r=\psi(\tilde{r}, \tau) l^{3 / 2}$, the GP equation (1) becomes

$$
\left[-\frac{\partial^{2}}{\partial r^{2}}+r^{2}+\aleph\left|\frac{\varphi(r ; t)}{r}\right|^{2}-\mathrm{i} \frac{\partial}{\partial t}\right] \varphi(r ; t)=0,
$$

where $\aleph=8 \pi N a / l$ with normalization (3). These three sets of dimensionless GP equations have been widely used in the literature and will be considered here. Equations (2), (4), and (5) allow stationary solutions $\varphi(r ; t) \equiv \varphi(r) \exp (-\mathrm{i} \mu t)$ where $\mu$ is the chemical potential. The boundary conditions for the solution of these equations are $\varphi(0, t)=0$ and $\lim _{r \rightarrow \infty} \varphi(r, t)=0$ [49].

\subsection{Anisotropic GP equation in 3D}

The three-dimensional trap potential is given by $V(\mathbf{r})=\frac{1}{2} m \omega^{2}\left(\nu^{2} \bar{x}^{2}+\kappa^{2} \bar{y}^{2}+\lambda^{2} \bar{z}^{2}\right)$, where $\omega_{x} \equiv \nu \omega, \omega_{y} \equiv$ $\omega \kappa$, and $\omega_{z} \equiv \omega \lambda$ are the angular frequencies in the $x, y$ and $z$ directions, respectively, and $\mathbf{r} \equiv(\bar{x}, \bar{y}, \bar{z})$ is the radial vector. In terms of dimensionless variables $x=\sqrt{2} \bar{x} / l, y=\sqrt{2} \bar{y} / l, z=\sqrt{2} \bar{z} / l, t=\tau \omega, l=\sqrt{\hbar /(m \omega))}$, and $\varphi(x, y, z ; t)=\sqrt{l^{3} /(2 \sqrt{2})} \Psi(\mathbf{r} ; \tau)$, the GP equation (1) becomes

$$
\left[-\frac{\partial^{2}}{\partial x^{2}}-\frac{\partial^{2}}{\partial y^{2}}-\frac{\partial^{2}}{\partial z^{2}}+\frac{1}{4}\left(\nu^{2} x^{2}+\kappa^{2} y^{2}+\lambda^{2} z^{2}\right)+\aleph|\varphi(x, y, z ; t)|^{2}-\mathrm{i} \frac{\partial}{\partial t}\right] \varphi(x, y, z ; t)=0,
$$

with $\aleph=8 \sqrt{2} \pi a N / l$ and normalization

$$
\int_{-\infty}^{\infty} d x \int_{-\infty}^{\infty} d y \int_{-\infty}^{\infty} d z|\varphi(x, y, z ; t)|^{2}=1
$$

Similarly, using $x=\bar{x} / l, y=\bar{y} / l, z=\bar{z} / l, t=\tau \omega, l=\sqrt{\hbar /(m \omega)}$, and $\varphi(x, y, z ; t)=\sqrt{l^{3}} \Psi(\mathbf{r} ; \tau)$, the GP equation (1) becomes

$$
\left[-\frac{1}{2} \frac{\partial^{2}}{\partial x^{2}}-\frac{1}{2} \frac{\partial^{2}}{\partial y^{2}}-\frac{1}{2} \frac{\partial^{2}}{\partial z^{2}}+\frac{1}{2}\left(\nu^{2} x^{2}+\kappa^{2} y^{2}+\lambda^{2} z^{2}\right)+\aleph|\varphi(x, y, z ; t)|^{2}-\mathrm{i} \frac{\partial}{\partial t}\right] \varphi(x, y, z ; t)=0,
$$

with $\aleph=4 \pi a N / l$ and normalization (7). Now with scaling $t \rightarrow 2 t$, Eq. (8) can be rewritten as 


$$
\left[-\frac{\partial^{2}}{\partial x^{2}}-\frac{\partial^{2}}{\partial y^{2}}-\frac{\partial^{2}}{\partial z^{2}}+\left(\nu^{2} x^{2}+\kappa^{2} y^{2}+\lambda^{2} z^{2}\right)+\aleph|\varphi(x, y, z ; t)|^{2}-\mathrm{i} \frac{\partial}{\partial t}\right] \varphi(x, y, z ; t)=0,
$$

with $\aleph=8 \pi a N / l$. The boundary conditions for solution are $\lim _{x \rightarrow \pm \infty} \varphi(x, y, z ; t)=0, \lim _{y \rightarrow \pm \infty} \varphi(x, y, z ; t)=$ $0, \lim _{z \rightarrow \pm \infty} \varphi(x, y, z ; t)=0$ [49].

\subsection{Axially-symmetric GP equation in 3D}

In the special case of axial symmetry $(\nu=\kappa)$ Eqs. (6), (8) and (9) can be simplified considering $\mathbf{r} \equiv(\rho, z)$ where $\rho=\sqrt{x^{2}+y^{2}}$ is the radial coordinate and $z$ is the axial coordinate. Then Eq. (6) becomes

$$
\left[-\frac{\partial^{2}}{\partial \rho^{2}}-\frac{1}{\rho} \frac{\partial}{\partial \rho}-\frac{\partial^{2}}{\partial z^{2}}+\frac{1}{4}\left(\kappa^{2} \rho^{2}+\lambda^{2} z^{2}\right)+\aleph|\varphi(\rho, z ; t)|^{2}-\mathrm{i} \frac{\partial}{\partial t}\right] \varphi(\rho, z ; t)=0,
$$

with $\aleph=8 \sqrt{2} \pi a N / l$ and normalization $2 \pi \int_{0}^{\infty} \rho d \rho \int_{-\infty}^{\infty} d z|\varphi(\rho, z ; t)|^{2}=1$. Similarly, Eqs. (8) and (9) can be written as

$$
\left[-\frac{1}{2} \frac{\partial^{2}}{\partial \rho^{2}}-\frac{1}{2 \rho} \frac{\partial}{\partial \rho}-\frac{1}{2} \frac{\partial^{2}}{\partial z^{2}}+\frac{1}{2}\left(\kappa^{2} \rho^{2}+\lambda^{2} z^{2}\right)+\aleph|\varphi(\rho, z ; t)|^{2}-\mathrm{i} \frac{\partial}{\partial t}\right] \varphi(\rho, z ; t)=0,
$$

with $\aleph=4 \pi a N / l$ and

$$
\left[-\frac{\partial^{2}}{\partial \rho^{2}}-\frac{1}{\rho} \frac{\partial}{\partial \rho}-\frac{\partial^{2}}{\partial z^{2}}+\left(\kappa^{2} \rho^{2}+\lambda^{2} z^{2}\right)+\aleph|\varphi(\rho, z ; t)|^{2}-\mathrm{i} \frac{\partial}{\partial t}\right] \varphi(\rho, z ; t)=0,
$$

with $\aleph=8 \pi a N / l$. In this case $\varphi(\rho=0, z ; t)$ is not zero but a constant. Convenient boundary conditions for solution in this case are $\lim _{z \rightarrow \pm \infty} \varphi(\rho, z ; t)=0, \lim _{\rho \rightarrow \infty} \varphi(\rho, z ; t)=0$, and $\partial \varphi(\rho, z ; t) /\left.\partial \rho\right|_{\rho=0}=0[12]$.

\subsection{One-dimensional GP equation}

In case of an elongated cigar-shaped trap, which is essentially an axially-symmetric trap with strong transverse confinement, Eq. (6) reduces to a quasi one-dimensional form. This is achieved by assuming that the system remains confined to the ground state in the transverse direction. In this case the wave function of Eq. (6) can be written as $\varphi(x, y, z ; t)=\tilde{\varphi}(x ; t) \phi_{0}(y) \phi_{0}(z) \exp [-i(\lambda+\kappa) t / 2]$ with $\phi_{0}(y)=[\kappa /(2 \pi)]^{1 / 4} \exp \left(-\kappa y^{2} / 4\right)$ and $\phi_{0}(z)=[\lambda /(2 \pi)]^{1 / 4} \exp \left(-\lambda z^{2} / 4\right)$ the respective ground state wave functions in $y$ and $z$ directions. Using

this ansatz in Eq. (6), multiplying by $\phi_{0}(y) \phi_{0}(z)$, integrating over $y$ and $z$, dropping the tilde over $\varphi$, and setting $\nu=1$ we obtain

$$
\left[-\frac{\partial^{2}}{\partial x^{2}}+\frac{x^{2}}{4}+\aleph|\varphi(x ; t)|^{2}-\mathrm{i} \frac{\partial}{\partial t}\right] \varphi(x ; t)=0,
$$

with $\aleph=2 a N \sqrt{2 \lambda \kappa} / l$ and normalization

$$
\int_{-\infty}^{\infty} d x|\varphi(x ; t)|^{2}=1
$$

Instead if we employ $\varphi(x, y, z ; t)=\tilde{\varphi}(x ; t) \phi_{0}(y) \phi_{0}(z) \exp [-i(\lambda+\kappa) t / 2]$ with $\phi_{0}(y)=(\kappa / \pi)^{1 / 4} \exp \left(-\kappa y^{2} / 2\right)$ and $\phi_{0}(z)=(\lambda / \pi)^{1 / 4} \exp \left(-\lambda z^{2} / 2\right)$ in Eq. (8), in a similar fashion we obtain

$$
\left[-\frac{1}{2} \frac{\partial^{2}}{\partial x^{2}}+\frac{x^{2}}{2}+\aleph|\varphi(x ; t)|^{2}-\mathrm{i} \frac{\partial}{\partial t}\right] \varphi(x ; t)=0,
$$

with $\aleph=2 a N \sqrt{\lambda \kappa} / l$ and normalization (14). Now with scaling $t \rightarrow 2 t$ Eq. (15) can be rewritten as

$$
\left[-\frac{\partial^{2}}{\partial x^{2}}+x^{2}+\aleph|\varphi(x ; t)|^{2}-\mathrm{i} \frac{\partial}{\partial t}\right] \varphi(x ; t)=0,
$$

with $\aleph=4 a N \sqrt{\lambda \kappa} / l$ and normalization (14). For numerical solution we take $\lim _{x \rightarrow \pm \infty} \varphi(x, t)=0$. 


\subsection{Anisotropic GP equation in 2D}

In case of a disk-shaped trap, which is essentially an anisotropic trap in two dimensions with strong axial binding Eq. (6) reduces to a two-dimensional form. This is achieved by assuming that the system remains confined to the ground state in the axial direction. In this case the wave function of Eq. (6) can be written as $\varphi(x, y, z ; t)=\tilde{\varphi}(x, y ; t) \phi_{0}(z) \exp [-i \lambda t / 2]$ with $\phi_{0}(z)=[\lambda /(2 \pi)]^{1 / 4} \exp \left(-\lambda z^{2} / 4\right)$ the ground state wave function in $z$ direction. Using this ansatz in Eq. (6), multiplying by $\phi_{0}(z)$, integrating over $z$, dropping the tilde over $\varphi$ and setting $\nu=1$ we obtain

$$
\left[-\frac{\partial^{2}}{\partial x^{2}}-\frac{\partial^{2}}{\partial y^{2}}+\frac{x^{2}+\kappa y^{2}}{4}+\aleph|\varphi(x, y ; t)|^{2}-\mathrm{i} \frac{\partial}{\partial t}\right] \varphi(x, y ; t)=0,
$$

now with $\aleph=4 a N \sqrt{2 \pi \lambda} / l$ and normalization

$$
\int_{-\infty}^{\infty} d x \int_{-\infty}^{\infty} d y|\varphi(x, y ; t)|^{2}=1
$$

Instead if we use in Eq. (8) $\phi(x, y, z ; t)=\tilde{\varphi}(x, y ; t) \phi_{0}(z) \exp [-i \lambda t / 2]$ with $\phi_{0}(z)=[\lambda / \pi]^{1 / 4} \exp \left(-\lambda z^{2} / 2\right)$, then in a similar fashion we obtain

$$
\left[-\frac{1}{2} \frac{\partial^{2}}{\partial x^{2}}-\frac{1}{2} \frac{\partial^{2}}{\partial y^{2}}+\frac{x^{2}+\kappa y^{2}}{2}+\aleph|\varphi(x, y ; t)|^{2}-\mathrm{i} \frac{\partial}{\partial t}\right] \varphi(x, y ; t)=0,
$$

now with $\aleph=2 a N \sqrt{2 \pi \lambda} / l$ and normalization (18). Finally, with scaling $t \rightarrow 2 t$, Eq. (19) can be written as

$$
\left[-\frac{\partial^{2}}{\partial x^{2}}-\frac{\partial^{2}}{\partial y^{2}}+x^{2}+\kappa y^{2}+\aleph|\varphi(x, y ; t)|^{2}-\mathrm{i} \frac{\partial}{\partial t}\right] \varphi(x, y ; t)=0
$$

with $\aleph=4 a N \sqrt{2 \pi \lambda} / l$ and normalization (18). For numerical solution we take $\lim _{x \rightarrow \pm \infty} \varphi(x, y, t)=0$ and $\lim _{y \rightarrow \pm \infty} \varphi(x, y, t)=0$.

\subsection{Circularly-symmetric GP equation in 2D}

In the special case of circular symmetry the equations of Sec. 2.5 can be written in one-dimensional form. In this case $\kappa=1$, and we introduce the radial variable $\mathbf{r} \equiv(x, y)$, and rewrite the wave function as $\varphi(r)$. Then the GP equation (17) become

$$
\left[-\frac{\partial^{2}}{\partial r^{2}}-\frac{1}{r} \frac{\partial}{\partial r}+\frac{r^{2}}{4}+\aleph|\varphi(r ; t)|^{2}-\mathrm{i} \frac{\partial}{\partial t}\right] \varphi(r ; t)=0 .
$$

The normalization of the wave function is $2 \pi \int_{0}^{\infty} d r r|\varphi(r ; t)|^{2}=1$.

In the circularly-symmetric case Eq. (19) becomes

$$
\left[-\frac{1}{2} \frac{\partial^{2}}{\partial r^{2}}-\frac{1}{2 r} \frac{\partial}{\partial r}+\frac{1}{2} r^{2}+\aleph|\varphi(r ; t)|^{2}-\mathrm{i} \frac{\partial}{\partial t}\right] \varphi(r ; t)=0 .
$$

Finally, Eq. (20) can be written as

$$
\left[-\frac{\partial^{2}}{\partial r^{2}}-\frac{1}{r} \frac{\partial}{\partial r}+r^{2}+\aleph|\varphi(r ; t)|^{2}-\mathrm{i} \frac{\partial}{\partial t}\right] \varphi(r ; t)=0 .
$$

The convenient boundary condition in this case is $\lim _{r \rightarrow \infty} \varphi(r ; t)=0$ and $d \varphi(r ; t) /\left.d r\right|_{r=0}=0$ [12].

In this section we have exhibited GP equations for different trap symmetries. In the next section we illustrate the Crank-Nicolson method for the GP equation in one space variable, which is then extended to other types of equations in Sec. 4. 


\section{Split-Step Crank-Nicolson Method for the GP Equation in one Space Variable}

\subsection{The GP Equation in the $1 D$ and radially-symmetric $3 D$ cases}

To introduce the Crank-Nicolson Method [49-51] for GP equation we consider first the one-dimensional case of Sec. 2.4. The nonlinear GP equation (13) in this case can be expressed in the following form:

$$
\begin{aligned}
\mathrm{i} \frac{\partial}{\partial t} \varphi(x ; t) & =\left[-\frac{\partial^{2}}{\partial x^{2}}+\frac{x^{2}}{4}+\aleph|\varphi(x ; t)|^{2}\right] \varphi(x ; t), \\
& \equiv H \varphi(x ; t)
\end{aligned}
$$

where the Hamiltonian $H$ contains the different linear and nonlinear terms including the spatial derivative. (The spherically-symmetric GP equation in 3D has a similar structure and can be treated similarly.) We solve this equation by time iteration [49-51]. A given trial input solution is propagated in time over small time steps until a stable final solution is reached. The GP equation is discretized in space and time using the finite difference scheme. This procedure results in a set of algebraic equations which can be solved by time iteration using an input solution consistent with the known boundary condition. In the present split-step method [50] this iteration is conveniently done in few steps by breaking up the full Hamiltonian into different derivative and non-derivative parts.

\subsubsection{Real-time propagation}

The time iteration is performed by splitting $H$ into two parts: $H=H_{1}+H_{2}$, with

$$
\begin{aligned}
& H_{1}=\left[\frac{x^{2}}{4}+\aleph|\varphi(x ; t)|^{2}\right], \\
& H_{2}=-\frac{\partial^{2}}{\partial x^{2}} .
\end{aligned}
$$

Essentially we split Eq. (24) into

$$
\begin{aligned}
\mathrm{i} \frac{\partial}{\partial t} \varphi(x ; t) & =\left[\frac{x^{2}}{4}+\aleph|\varphi(x ; t)|^{2}\right] \varphi(x ; t) \equiv H_{1} \varphi(x ; t) \\
\mathrm{i} \frac{\partial}{\partial t} \varphi(x ; t) & =-\frac{\partial^{2}}{\partial x^{2}} \varphi(x ; t) \equiv H_{2} \varphi(x ; t)
\end{aligned}
$$

We first solve Eq. (27) with a initial value $\varphi\left(x ; t_{0}\right)$ at $t=t_{0}$ to obtain an intermediate solution at $t=t_{0}+\Delta$, where $\Delta$ is the time step. Then this intermediate solution is used as initial value to solve Eq. (28) yielding the final solution at $t=t_{0}+\Delta$ as $\varphi\left(x ; t_{0}+\Delta\right)$. This procedure is repeated $n$ times to get the final solution at a given time $t_{\text {final }}=t_{0}+n \Delta$.

The time variable is discretized as $t_{n}=n \Delta$ where $\Delta$ is the time step. The solution is advanced first over the time step $\Delta$ at time $t_{n}$ by solving the GP equation (24) with $H=H_{1}$ to produce an intermediate solution $\varphi^{n+1 / 2}$ from $\varphi^{n}$, where $\varphi^{n}$ is the discretized wave function at time $t_{n}$. As there is no derivative in $H_{1}$, this propagation is performed essentially exactly for small $\Delta$ through the operation

$$
\varphi^{n+1 / 2}=\bigcirc_{\text {nd }}\left(H_{1}\right) \varphi^{n} \equiv e^{-\mathrm{i} \Delta H_{1}} \varphi^{n},
$$

where $\bigcirc_{\text {nd }}\left(H_{1}\right)$ denotes time-evolution operation with $H_{1}$ and the suffix 'nd' denotes non-derivative. Next we perform the time propagation corresponding to the operator $H_{2}$ numerically by the semi-implicit CrankNicolson scheme (described below) [49]:

$$
\frac{\varphi^{n+1}-\varphi^{n+1 / 2}}{-\mathrm{i} \Delta}=\frac{1}{2} H_{2}\left(\varphi^{n+1}+\varphi^{n+1 / 2}\right) .
$$

The formal solution to $(30)$ is

$$
\varphi^{n+1}=\bigcirc_{\mathrm{CN}}\left(H_{2}\right) \varphi^{n+1 / 2} \equiv \frac{1-\mathrm{i} \Delta H_{2} / 2}{1+\mathrm{i} \Delta H_{2} / 2} \varphi^{n+1 / 2},
$$


which combined with Eq. (29) yields

$$
\varphi^{n+1}=\bigcirc_{\mathrm{CN}}\left(H_{2}\right) \bigcirc_{\mathrm{nd}}\left(H_{1}\right) \varphi^{n}
$$

where $\bigcirc_{\mathrm{CN}}$ denotes time-evolution operation with $\mathrm{H}_{2}$ and the suffix 'CN' refers to the Crank-Nicolson algorithm. Operation $\bigcirc_{\mathrm{CN}}$ is used to propagate the intermediate solution $\varphi^{n+1 / 2}$ by time step $\Delta$ to generate the solution $\varphi^{n+1}$ at the next time step $t_{n+1}=(n+1) \Delta$.

The advantage of the above split-step method with small time step $\Delta$ is due to the following three factors [50,51]. First, all iterations conserve normalization of the wave function. Second, the error involved in splitting the Hamiltonian is proportional to $\Delta^{2}$ and can be neglected and the method preserves the symplectic structure of the Hamiltonian formulation. Finally, as a major part of the Hamiltonian including the nonlinear term is treated fairly accurately without mixing with the delicate Crank-Nicolson propagation, the method can deal with an arbitrarily large nonlinear term and lead to stable and accurate converged result.

Now we describe explicitly the semi-implicit Crank-Nicolson algorithm. The GP equation is mapped onto $N_{x}$ one-dimensional spatial grid points in $x$. Equation (24) is discretized with $H=H_{2}$ of (26) by the following Crank-Nicolson scheme [49-51]:

$$
\frac{\mathrm{i}\left(\varphi_{i}^{n+1}-\varphi_{i}^{n+1 / 2}\right)}{\Delta}=-\frac{1}{2 h^{2}}\left[\left(\varphi_{i+1}^{n+1}-2 \varphi_{i}^{n+1}+\varphi_{i-1}^{n+1}\right)+\left(\varphi_{i+1}^{n+1 / 2}-2 \varphi_{i}^{n+1 / 2}+\varphi_{i-1}^{n+1 / 2}\right)\right],
$$

where for the spherically-symmetric Eq. (2) $\varphi_{i}^{n}=\varphi\left(x_{i} ; t_{n}\right)$ refers to $x \equiv x_{i}=i h, i=0,1,2, \ldots, N_{x}$ and $h$ is the space step. In the case of the 1D Eq. (13), we choose $x \equiv x_{i}=-N_{x} h / 2+i h, i=0,1,2, \ldots, N_{x}$. (The choice - even $N_{x}$ - has the advantage of taking an equal number of space points on both sides of $x=0$ in the $1 \mathrm{D}$ case setting the point $N_{x} / 2$ at $x=0$.) Equation (33) is the explicit form of the formal Eq. (30). This scheme is constructed by approximating $\partial / \partial t$ by a two-point formula connecting the present $(n+1 / 2)$ to future $(n+1)$. The spatial partial derivative $\partial^{2} / \partial x^{2}$ is approximated by a three-point formula averaged over the present and the future time grid points. This procedure results in a series of tridiagonal sets of equations (33) in $\varphi_{i+1}^{n+1}, \varphi_{i}^{n+1}$, and $\varphi_{i-1}^{n+1}$ at time $t_{n+1}$, which are solved using the proper boundary conditions.

The Crank-Nicolson scheme (33) possesses certain properties worth mentioning [50,51]. The error in this scheme is both second order in space and time steps so that for small $\Delta$ and $h$ the error is negligible. This scheme is also unconditionally stable [51]. The boundary condition at infinity is preserved for small values of $\Delta / h^{2}[51]$.

The tridiagonal equations emerging from Eq. (33) are written explicitly as [49]

$$
A_{i}^{-} \varphi_{i-1}^{n+1}+A_{i}^{0} \varphi_{i}^{n+1}+A_{i}^{+} \varphi_{i+1}^{n+1}=b_{i},
$$

where

$$
b_{i}=\frac{\mathrm{i} \Delta}{2 h^{2}}\left(\varphi_{i+1}^{n+1 / 2}-2 \varphi_{i}^{n+1 / 2}+\varphi_{i-1}^{n+1 / 2}\right)+\varphi_{i}^{n+1 / 2},
$$

and $A_{i}^{-}=A_{i}^{+}=-\mathrm{i} \Delta /\left(2 h^{2}\right), A_{i}^{0}=1+\mathrm{i} \Delta / h^{2}$. All quantities in $b_{i}$ refer to time step $t_{n+1 / 2}$ and are considered known. The only unknowns in Eq. (34) are the wave forms $\varphi_{i \pm 1}^{n+1}$ and $\varphi_{i}^{n+1}$ at time step $t_{n+1}$. To solve Eq. (34), we assume the one-term forward recursion relation

$$
\varphi_{i+1}^{n+1}=\alpha_{i} \varphi_{i}^{n+1}+\beta_{i},
$$

where $\alpha_{i}$ and $\beta_{i}$ are coefficients to be determined. Substituting Eq. (36) in Eq. (34) we obtain

$$
A_{i}^{-} \varphi_{i-1}^{n+1}+A_{i}^{0} \varphi_{i}^{n+1}+A_{i}^{+}\left(\alpha_{i} \varphi_{i}^{n+1}+\beta_{i}\right)=b_{i},
$$

which leads to the solution

$$
\varphi_{i}^{n+1}=\gamma_{i}\left(A_{i}^{-} \varphi_{i-1}^{n+1}+A_{i}^{+} \beta_{i}-b_{i}\right),
$$

with

$$
\gamma_{i}=-1 /\left(A_{i}^{0}+A_{i}^{+} \alpha_{i}\right) .
$$

From Eqs. (36) and (38) we obtain the following backward recursion relations for the coefficients $\alpha_{i}$ and $\beta_{i}$

$$
\alpha_{i-1}=\gamma_{i} A_{i}^{-}, \quad \beta_{i-1}=\gamma_{i}\left(A_{i}^{+} \beta_{i}-b_{i}\right) .
$$


We shall use the recursion relations (38), (39) and (40) in a backward sweep of the lattice to determine $\alpha_{i}$ and $\beta_{i}$ for $i$ running from $N_{x}-2$ down to 0 . The initial values chosen are $\alpha_{N_{x}-1}=0, \beta_{N_{x}-1}=\varphi_{N_{x}}^{n+1}$. This ensures the correct value of $\varphi$ at the last lattice point. After determining the coefficients $\alpha_{i}, \beta_{i}$ and $\gamma_{i}$, we can use the recursion relation (36) from $i=0$ to $N_{x}-1$ to determine the solution for the entire space range using the starting value $\varphi_{0}^{n+1}(=0)$ known from the boundary conditions. The value at the last lattice point is also taken to be known $(=0)$. Thus we have determined the solution by using two sets of recursion relations across the lattice each involving about $N_{x}$ operations.

In the numerical implementation of $\mathrm{CN}$ real-time propagation the initial state at $t=0$ is usually chosen to be the analytically known solution of the harmonic potential with zero nonlinearity: $\aleph=0$. In the course of time iteration the nonlinearity is slowly introduced until the desired final nonlinearity is attained. This procedure will lead us to the final solution of the problem.

\subsubsection{Imaginary-time propagation}

Although, real-time propagation as described above has many advantages, in this approach one has to deal with complex variables for a complex wave function for non-stationary states. For stationary ground state the wave function is essentially real- and the imaginary-time propagation method dealing with real variables seems to be convenient. In this approach time $t$ is replaced by an imaginary quantity $t=-\mathrm{i} \bar{t}$ and Eq. (24) now becomes

$$
\begin{aligned}
-\frac{\partial}{\partial \bar{t}} \varphi(x ; \bar{t}) & =\left[-\frac{\partial^{2}}{\partial x^{2}}+\frac{x^{2}}{4}+\aleph|\varphi(x ; \bar{t})|^{2}\right] \varphi(x ; \bar{t}), \\
& \equiv H \varphi(x ; \bar{t})
\end{aligned}
$$

In this equation $\bar{t}$ is just a mathematical parameter.

From Eq. (41) we see that an eigenstate $\varphi_{i}$ of eigenvalue $E_{i}$ of $H$ satisfying $H \varphi_{i}=E_{i} \varphi_{i}$ behaves under imaginary-time propagation as $\partial \varphi_{i}(\bar{t}) / \partial \bar{t}=-E_{i} \varphi_{i}(\bar{t})$, so that $\varphi_{i}(\bar{t})=\exp \left(-E_{i} \bar{t}\right) \varphi_{i}(0)$. Hence, if we start with as arbitrary initial $\varphi(x ; \bar{t})$ which can be taken as a linear combination of all eigenfunctions of $H$, then upon imaginary-time propagation all the eigenfunctions will decay exponentially with time. However, all the excited states with larger $E_{i}$ will decay exponentially faster compared to the ground state with the smallest eigenvalue. Consequently, after some time only the ground state survives. As all the states are decaying with time during imaginary-time propagation, we need to multiply the wave function by a number greater than unity to preserve its normalization so that the solution does not go to zero.

The imaginary-time iteration is performed by splitting $H$ into two parts as before: $H=H_{1}+H_{2}$, with $H_{1}$ and $H_{2}$ given by Eqs. (25) and (26). It is realized that the entire analysis of Sec. 3.1.1 remains valid provided we replace i by 1 in Eq. (29) and by -1 in the remaining equations. However, there appears one trouble. The CN real-time propagation preserves the normalization of the wave function, whereas the CN imaginarytime propagation does not preserve the normalization. This problem can be circumvented by restoring the normalization of the wave function after each operation of Crank-Nicolson propagation. Once this is done the imaginary-time propagation method for stationary ground state problems yields very accurate result at low computational cost.

Compared to the real-time propagation method, the imaginary-time propagation method is very robust. The initial solution in the imaginary-time method could be any reasonable solution and not the analytically known solution of a related problem as in the real-time method. Also, the full nonlinearity can be added in a small number of time steps or even in a single step and not in a large number of steps as in the real-time method. In the programs using the imaginary-time method we include the nonlinearity in a single step. These two added features together with the use of real algorithm make the imaginary-time propagation method very accurate with quick convergence for stationary ground states as we shall see below.

\subsection{The GP Equation in the circularly-symmetric 2D case}

The Crank-Nicolson discretization for real- and imaginary-time propagation for the circularly-symmetric GP equation (21) is performed in a similar fashion as for Eq. (24), apart from the difference that here 
we also have a first derivative in space variable in addition to the second derivative. Another difference is that the wave function is not zero at $r=0$. In the case of Eq. (24) we took the boundary condition as $\varphi(x ; t)=0$ at the boundaries. For the circularly-symmetric case, the convenient boundary conditions are $\lim _{r \rightarrow \infty} \varphi(r ; t)=0$ and $d \varphi(r ; t) /\left.d r\right|_{r=0}=0$.

We describe below the Crank-Nicolson discretization and the solution algorithm in this case for the following equation

$$
\left[-\frac{\partial^{2}}{\partial r^{2}}-\frac{1}{r} \frac{\partial}{\partial r}-\mathrm{i} \frac{\partial}{\partial t}\right] \varphi(r ; t)=0,
$$

required for the solution of Eq. (21). The remaining procedure is similar to that described in detail above in the one-dimensional case.

Equation (42) is discretized by the following Crank-Nicolson scheme as in Eq. (33) [49-51]:

$$
\begin{array}{r}
\frac{\mathrm{i}\left(\varphi_{i}^{n+1}-\varphi_{i}^{n+1 / 2}\right)}{\Delta}=-\frac{1}{2 h^{2}}\left[\left(\varphi_{i+1}^{n+1}-2 \varphi_{i}^{n+1}+\varphi_{i-1}^{n+1}\right)+\left(\varphi_{i+1}^{n+1 / 2}-2 \varphi_{i}^{n+1 / 2}+\varphi_{i-1}^{n+1 / 2}\right)\right] \\
-\frac{1}{4 r_{i} h}\left[\left(\varphi_{i+1}^{n+1}-\varphi_{i-1}^{n+1}\right)+\left(\varphi_{i+1}^{n+1 / 2}-\varphi_{i-1}^{n+1 / 2}\right)\right],
\end{array}
$$

where again $\varphi_{i}^{n}=\varphi\left(r_{i} ; t_{n}\right), r \equiv r_{i}=i h, i=0,1,2, \ldots, N_{r}$ and $h$ is the space step. This scheme is constructed by approximating $\partial / \partial x$ by a two-point formula averaged over present and future time grid points. The discretization of the first-order time and second-order space derivatives is done as in Eq. (33). This procedure results in the tridiagonal sets of equations (43) in $\varphi_{i+1}^{n+1}, \varphi_{i}^{n+1}$, and $\varphi_{i-1}^{n+1}$ at time $t_{n+1}$, which are solved using the proper boundary conditions.

The tridiagonal equations emerging from Eq. (43) are written explicitly as

$$
A_{i}^{-} \varphi_{i-1}^{n+1}+A_{i}^{0} \varphi_{i}^{n+1}+A_{i}^{+} \varphi_{i+1}^{n+1}=b_{i}
$$

where

$$
b_{i}=\frac{\mathrm{i} \Delta}{2 h^{2}}\left(\varphi_{i+1}^{n+1 / 2}-2 \varphi_{i}^{n+1 / 2}+\varphi_{i-1}^{n+1 / 2}\right)+\varphi_{i}^{n+1 / 2}+\frac{\mathrm{i} \Delta}{4 r_{i} h}\left(\varphi_{i+1}^{n+1 / 2}-\varphi_{i-1}^{n+1 / 2}\right),
$$

and $A_{i}^{-}=\mathrm{i} \Delta\left[1 /\left(4 h r_{i}\right)-1 /\left(2 h^{2}\right)\right], A_{i}^{+}=-\mathrm{i} \Delta\left[1 /\left(4 h r_{i}\right)+1 /\left(2 h^{2}\right)\right], A_{i}^{0}=1+\mathrm{i} \Delta / h^{2}$. All quantities in $b_{i}$ refer to time step $t_{n+1 / 2}$ and are considered known. The only unknowns in Eq. (44) are the wave forms $\varphi_{i \pm 1}^{n+1}$ and $\varphi_{i}^{n+1}$ at time step $t_{n+1}$. To solve Eq. (44), we assume the one-term backward recursion relation

$$
\varphi_{i-1}^{n+1}=\alpha_{i} \varphi_{i}^{n+1}+\beta_{i}
$$

where $\alpha_{i}$ and $\beta_{i}$ are coefficients to be determined. Substituting Eq. (46) in Eq. (44) we obtain

$$
A_{i}^{+} \varphi_{i+1}^{n+1}+A_{i}^{0} \varphi_{i}^{n+1}+A_{i}^{-}\left(\alpha_{i} \varphi_{i}^{n+1}+\beta_{i}\right)=b_{i},
$$

which leads to the solution

$$
\varphi_{i}^{n+1}=\gamma_{i}\left(A_{i}^{+} \varphi_{i+1}^{n+1}+A_{i}^{-} \beta_{i}-b_{i}\right)
$$

with

$$
\gamma_{i}=-1 /\left(A_{i}^{0}+A_{i}^{-} \alpha_{i}\right)
$$

From Eqs. (46) and (48) we obtain the following forward recursion relations for the coefficients $\alpha_{i}$ and $\beta_{i}$

$$
\alpha_{i+1}=\gamma_{i} A_{i}^{+}, \quad \beta_{i+1}=\gamma_{i}\left(A_{i}^{-} \beta_{i}-b_{i}\right) .
$$

We shall use the recursion relations (48), (49) and (50) in a forward sweep of the lattice to determine $\alpha_{i}$ and $\beta_{i}$ for $i$ running from 1 to $N_{r}-1$. The initial values chosen are $\alpha_{1}=1, \beta_{1}=0$. This ensures the correct value of the space derivative of $\varphi(r ; t)=0$ at $r=0$. After determining the coefficients $\alpha_{i}, \beta_{i}$ and $\gamma_{i}$, we can use the recursion relation (46) from $i=N_{r}$ to 1 to determine the solution for the entire space range using the starting value $\varphi^{n+1}\left(N_{r}\right)(=0)$ known from the boundary condition. Thus we have determined the solution by using two sets of recursion relations across the lattice each involving about $N_{r}$ operations. 


\subsection{Chemical potential}

For stationary states the wave functions for the 1D case have the trivial time dependence $\varphi(x ; t) \equiv$ $\hat{\varphi}(x) \exp (-\mathrm{i} \mu t)$, where $\mu$ is the chemical potential. Substituting this condition in Eq. (13) we obtain

$$
\left[-\frac{d^{2}}{d x^{2}}+\frac{x^{2}}{4}+\aleph \hat{\varphi}^{2}(x)\right] \hat{\varphi}(x)=\mu \hat{\varphi}(x) .
$$

Assuming that the wave form is normalized to unity $\int_{-\infty}^{\infty} \hat{\varphi}^{2}(x) d x=1$, the chemical potential can be calculated from the following expression obtained by multiplying Eq. (51) by $\hat{\varphi}(x)$ and integrating over all space

$$
\mu=\int_{-\infty}^{\infty}\left[\left(\frac{d \hat{\varphi}(x)}{d x}\right)^{2}+\hat{\varphi}^{2}(x)\left(\frac{x^{2}}{4}+\aleph \hat{\varphi}^{2}(x)\right)\right] d x,
$$

where the second derivative has been simplified by an integration by parts.

All the programs also calculate the many-body energy, which is of interest. The analytical expression for energy is the same as that of the chemical potential but with the nonlinear term multiplied by $1 / 2$, e.g., [1]

$$
E=\int_{-\infty}^{\infty}\left[\left(\frac{d \hat{\varphi}(x)}{d x}\right)^{2}+\hat{\varphi}^{2}(x)\left(\frac{x^{2}}{4}+\frac{\aleph}{2} \hat{\varphi}^{2}(x)\right)\right] d x .
$$

The programs will write the value of energy as output. However, we shall not study or tabulate the results for energy and we shall not write the explicit algebraic expression of energy in the case of other trap symmetries.

The GP equation (2) with spherically-symmetric potential is also an one-variable equation quite similar in structure to the one-dimensional equation (13) considered above. Hence the entire analysis of Secs. 3.1.1, 3.1 .2 , and 3.3 will be applicable in this case with $\varphi(x ; t)$ replaced by $\varphi(r ; t) / r$ in the nonlinear term. Now if we consider stationary states of the form $\varphi(r, t) \equiv \hat{\varphi}(r) \exp (-\mathrm{i} \mu t / \hbar)$, the expression for the chemical potential becomes

$$
\mu=4 \pi \int_{0}^{\infty}\left[\left(\frac{d \hat{\varphi}(r)}{d r}\right)^{2}+\hat{\varphi}^{2}(r)\left(\frac{r^{2}}{4}+\aleph \frac{\hat{\varphi}^{2}(r)}{r^{2}}\right)\right] d r .
$$

We shall use Eqs. (52) and (54) for the calculation of chemical potential from Eqs. (13) and (2). The energy will be calculated from Eq. (53). The expressions for chemical potential in other cases can be written down in a straight-forward fashion.

\section{Split-Step Crank-Nicolson method in two and three space variables}

\subsection{Anisotropic GP equation in 2D}

In this case the GP equation (17) can be written as

$$
\begin{aligned}
\mathrm{i} \frac{\partial}{\partial t} \varphi(x, y ; t) & =\left[-\frac{\partial^{2}}{\partial x^{2}}-\frac{\partial^{2}}{\partial y^{2}}+\frac{1}{4}\left(x^{2}+\kappa^{2} y^{2}\right)+\aleph|\varphi(x, y ; t)|^{2}\right] \varphi(x, y ; t) \\
& \equiv H \varphi(x, y ; t),
\end{aligned}
$$

with $\aleph=4 \sqrt{2 \pi \lambda} N a / l$. The Hamiltonian $H$ can be conveniently broken into three pieces $H=H_{1}+H_{2}+H_{3}$, where

$$
\begin{aligned}
& H_{1}=\frac{1}{4}\left(x^{2}+\kappa^{2} y^{2}\right)+\aleph|\varphi(x, y ; t)|^{2}, \\
& H_{2}=-\frac{\partial^{2}}{\partial x^{2}}, \quad H_{3}=-\frac{\partial^{2}}{\partial y^{2}} .
\end{aligned}
$$

Now we adopt a policy quite similar to that elaborated in Sec. 3.1.1 where the Hamiltonian was broken into two parts and where the time propagation over time step $\Delta$ using the two parts were carried out alternatively. 
The same procedure will be adopted in the present case where we perform the time propagation using the pieces $H_{1}, H_{2}$, and $H_{3}$ of the Hamiltonian successively in three independent time sub-steps $\Delta$ to complete a single time evolution over time step $\Delta$ of the entire GP Hamiltonian $H$. The time propagation over $H_{1}$ is performed as in Eq. (29) and those over $H_{2}$ and $H_{3}$ as in Eqs. (31) and (33). The chemical potential in this case for the stationary state $\varphi(x, y ; t) \equiv \hat{\varphi}(x, y) \exp (-\mathrm{i} \mu t)$ can be written as

$$
\mu=\int_{-\infty}^{\infty} d x \int_{-\infty}^{\infty} d y\left[\left(\frac{\partial \hat{\varphi}}{\partial x}\right)^{2}+\left(\frac{\partial \hat{\varphi}}{\partial y}\right)^{2}+\hat{\varphi}^{2}\left(\frac{x^{2}+\kappa^{2} y^{2}}{4}+\aleph \hat{\varphi}^{2}\right)\right],
$$

where we have performed integrations by parts to obtain this form for the chemical potential in terms of first derivatives only.

\subsection{Axially-symmetric GP equation in 3D}

In this case the GP equation (10) can be written as

$$
\begin{aligned}
\mathrm{i} \frac{\partial}{\partial t} \varphi(x, y ; t) & =\left[-\frac{\partial^{2}}{\partial \rho^{2}}-\frac{1}{\rho} \frac{\partial}{\partial \rho}-\frac{\partial^{2}}{\partial z^{2}}+\frac{1}{4}\left(\kappa^{2} \rho^{2}+\lambda^{2} z^{2}\right)+\aleph|\varphi(\rho, z ; t)|^{2}\right] \varphi(\rho, z ; t) \\
& \equiv H \varphi(\rho, z ; t) .
\end{aligned}
$$

The Hamiltonian $H$ can be conveniently broken into three pieces $H=H_{1}+H_{2}+H_{3}$, where

$$
\begin{aligned}
& H_{1}=\frac{1}{4}\left(\rho^{2}+\lambda^{2} z^{2}\right)+\aleph|\varphi(\rho, z ; t)|^{2}, \\
& H_{2}=-\frac{\partial^{2}}{\partial \rho^{2}}-\frac{1}{\rho} \frac{\partial}{\partial \rho}, \quad H_{3}=-\frac{\partial^{2}}{\partial z^{2}} .
\end{aligned}
$$

Now we adopt a policy quite similar to that elaborated in Sec. 4.1 where the Hamiltonian was broken into three parts and where the time propagation over time step $\Delta$ using the three parts were carried out alternatively. The time propagation over $H_{1}$ is performed as in Eq. (29) and those over $H_{2}$ and $H_{3}$ as in Eqs. (43) and (33). The chemical potential in this case for the stationary state $\varphi(\rho, z ; t) \equiv \hat{\varphi}(\rho, z) \exp (-\mathrm{i} \mu t)$ can be written as

$$
\mu=2 \pi \int_{0}^{\infty} \rho d \rho \int_{-\infty}^{\infty} d z\left[\left(\frac{\partial \hat{\varphi}}{\partial \rho}\right)^{2}+\left(\frac{\partial \hat{\varphi}}{\partial z}\right)^{2}+\hat{\varphi}^{2}\left(\frac{\kappa^{2} \rho^{2}+\lambda^{2} z^{2}}{4}+\aleph \hat{\varphi}^{2}\right)\right],
$$

where we have again used integrations by parts to simplify the final expression.

\subsection{Anisotropic GP equation in 3D}

In this case the GP equation (6) can be written as

$$
\begin{aligned}
\mathrm{i} \frac{\partial}{\partial t} \varphi(x, y, z ; t) & =\left[-\frac{\partial^{2}}{\partial x^{2}}-\frac{\partial^{2}}{\partial y^{2}}-\frac{\partial^{2}}{\partial z^{2}}+\frac{1}{4}\left(\nu^{2} x^{2}+\kappa^{2} y^{2}+\lambda^{2} z^{2}\right)+\aleph|\varphi(x, y, z ; t)|^{2}\right] \varphi(x, y, z ; t) \\
& \equiv H \varphi(x, y, z ; t),
\end{aligned}
$$

with $\aleph=8 \sqrt{2} \pi a N / l$. The Hamiltonian $H$ can be conveniently broken into four pieces $H=H_{1}+H_{2}+H_{3}+H_{4}$, where

$$
\begin{aligned}
& H_{1}=\frac{1}{4}\left(\nu^{2} x^{2}+\kappa^{2} y^{2}+\lambda^{2} z^{2}\right)+\aleph|\varphi(x, y, z ; t)|^{2}, \\
& H_{2}=-\frac{\partial^{2}}{\partial x^{2}}, \quad H_{3}=-\frac{\partial^{2}}{\partial y^{2}}, \quad H_{4}=-\frac{\partial^{2}}{\partial z^{2}} .
\end{aligned}
$$

Now we adopt a policy quite similar to that elaborated in Sec. 3.1.1 where the Hamiltonian was broken into two parts and where the time propagation over time step $\Delta$ using the two parts were carried out alternatively. 
The same procedure will be adopted in the present case where we perform the time propagation using the pieces $H_{1}, H_{2}, H_{3}$ and $H_{4}$ of the Hamiltonian successively in four independent time sub-steps $\Delta$ to complete a single time evolution over time step $\Delta$ of the entire GP Hamiltonian $H$. The time propagation over $H_{1}$ is performed as in Eq. (29) and those over $H_{2}, H_{3}$ and $H_{4}$ as in Eqs. (31) and (33). The chemical potential in this case for the stationary state $\varphi(x, y, z ; t) \equiv \hat{\varphi}(x, y, z) \exp (-\mathrm{i} \mu t)$ can be written as

$$
\mu=\int_{-\infty}^{\infty} d x \int_{-\infty}^{\infty} d y \int_{-\infty}^{\infty} d z\left[\left(\frac{\partial \hat{\varphi}}{\partial x}\right)^{2}+\left(\frac{\partial \hat{\varphi}}{\partial y}\right)^{2}+\left(\frac{\partial \hat{\varphi}}{\partial z}\right)^{2}+\hat{\varphi}^{2}\left(\frac{\nu^{2} x^{2}+\kappa^{2} y^{2}+\lambda^{2} z^{2}}{4}+\aleph \hat{\varphi}^{2}\right)\right],
$$

where we have again used integrations by parts to simplify the final expression.

\section{Description of Numerical Programs}

\subsection{GP equation in one space variable}

In this subsection we describe six Fortran codes involving GP equation in one space variable. These are programs for solving the 1D GP equation (program imagtime1d.F), the circularly-symmetric 2D GP equation (program imagtimecir.F) and the radially-symmetric 3D GP equation (program imagtimesph.F) using imaginary-time propagation. The similar routines using real-time propagation are realtime1d.F, realtimecir.F and realtimesph.F, respectively. These real- and imaginary-time routines in one space variable have similar structures. However, the wave function is real in imaginary-time propagation, whereas it is complex in real-time propagation. To accommodate this fact many variables in the real-time propagation routines are complex.

The principal variables employed in the MAIN program are: $\mathrm{N}=$ number of space mesh points, NSTP $=$ number of time iterations during which the nonlinearity is introduced in real-time propagation, (in imaginarytime propagation the nonlinearity is introduced in one step,) NPAS = number of subsequent time iterations with fixed nonlinearity, NRUN = number of final time iterations with (a) fixed nonlinearity in imaginarytime propagation and (b) modified nonlinearity to study dynamics in real-time propagation, $\mathrm{X}(\mathrm{I})=$ space mesh, $\mathrm{X} 2(\mathrm{I})=\mathrm{X}(\mathrm{I}) * \mathrm{X}(\mathrm{I}), \mathrm{V}(\mathrm{I})=$ potential, $\mathrm{CP}(\mathrm{I})=$ wave function at space point $\mathrm{X}(\mathrm{I}), \mathrm{G}, \mathrm{G} 0=$ coefficient of nonlinear term, $\mathrm{MU}=$ chemical potential, $\mathrm{EN}=$ energy, ZNORM = normalization of wave function, RMS $=$ rms size or radius, DX $=$ space step, DT $=$ time step, OPTION and XOP decide which equation to solve. Also important is the subroutine INITIALIZE, where the space mesh $\mathrm{X}(\mathrm{I})$, potential $\mathrm{V}(\mathrm{I})$, and the initial wave function $\mathrm{CP}(\mathrm{I})$, are calculated. (An advanced user may need to change the variables $\mathrm{V}(\mathrm{I})$ and $\mathrm{CP}(\mathrm{I})$ so as to adopt the program to solve a different equation with different nonlinearity.) The functions and variables not listed above are auxiliary variables, that the user should not need to modify.

Now we describe the function of the subroutines, which the user should not need to change. The subroutine NORM calculates by Simpson's rule the normalization of the wave function and sets the normalization to unity, the preassigned value. The real-time propagation preserves the normalization of the wave function and hence the subroutine NORM is not used during time propagation. The subroutines RAD and CHEM calculate the rms size (length or radius), and chemical potential (and energy) of the system. The subroutines $\mathrm{COEF}$ and LU together implement the time propagation with the spatial and temporal time derivative terms. The subroutine NU performs the time propagation with the nonlinear term and the potential. In the imaginary-time program the action of the subroutine LU does not preserve normalization and hence each time the subroutine LU is called, the subroutine NORM has to be called to set the normalization of the wave function back to unity. (This is not necessary in the real-time programs which preserve the norm.) The subroutine NONLIN calculates the nonlinear term. The subroutine DIFF calculates the space derivative of the wave function by Richardson's extrapolation formula needed for the computation of the chemical potential and energy. The function SIMP does the numerical space integrations with the Simpson's rule.

The programs implement the splitting method described in Sec. 3.1.1 and 3.2 and calculate the wave function, chemical potential, size, normalization, etc. The number of points in the one-dimensional space grid represented by the integer variable $\mathrm{N}$ has to be chosen consistent with space step DX such that the total space covered $\mathrm{N} \times \mathrm{DX}$ is significantly larger than the size of the condensate so that at the boundaries 
the wave function attains the asymptotic limits (e.g., the absolute value of the wave function or its space derivative becomes less than $10^{-10}$ or so for imaginary-time propagation and less than $10^{-7}$ for real-time propagation. Note that in the 1D and spherically-symmetric 3D problems we are using the asymptotic condition that the wave function is zero at both boundaries. In case of the circularly-symmetric 2D problem we use a mixed boundary condition, e.g., at origin the derivative of the wave function is zero and at infinity the wave function is zero.) In the imaginary-time routine the total space covered should be about 1.5 times the extension of the condensate. In the real-time routines, to obtain good precision the total space covered should be at least 2 times the extension of the condensate. This is because the imaginary-time routine is more precise and the solution attains its limiting asymptotic value at the boundary very rapidly as the total space covered is increased. The real-time routine is less accurate and one has to go to a larger distance before the solution or its derivative drops to zero. A couple of runs with a sufficiently large $\mathrm{N}$ and large DX are recommended to have an idea of the size/extension of the system. A smaller value of DX leads to a more accurate result provided an appropriate DT is chosen.

The Crank-Nicolson method, described, for example, by Eq. (33), is unconditionally stable for all $\Delta / h^{2}$. Nevertheless, for a numerical application to a specific problem one has to fix the time step DT $(=\Delta)$ and space step DX $(=h)$ for good convergence. Space and time steps are given in the MAIN program, as "DATA DX/0.0025D0/, DT/0.00002D0/" with correlated DX and DT values obtained by trial. (If the user wants to use other values of DX and DT, a set of correlated values obtained by trial is given in Table 1.) The total number of space points $\mathrm{N}$ in calculation has to be fixed in the line, e.g., "PARAMETER $(\mathrm{N}=6000, \mathrm{~N} 2=$ $\mathrm{N} / 2, \mathrm{NX}=\mathrm{N}-1$ )", in the MAIN program, so that the final wave function is within the space range and its value is negligibly small at the boundaries. The total numbers of time iterations are fixed in the line, e.g., "PARAMETER $($ NSTP $=500000$, NPAS $=10000$, NRUN $=20000) "$, in the MAIN program as described below in Sec. 5.3.

The integer parameter OPTION should be set 1, 2 or 3 in the MAIN routine for solution of equations of type (5), (4), or (2), [or for solution of equations of type (16), (15), or (13),] respectively. The difference between these three types of equations is in the values of the coefficients in the first two terms. The nonlinear term calculated in the subroutine NONLIN is of the form $|\varphi(r, t)|^{2}$ with coefficient G. If a different type of nonlinear term (with different functional dependence on the wave function) is to be introduced, it should be done in the subroutine NONLIN. Otherwise, this subroutine should not be changed. A different type of nonlinear term is appropriate for a Fermi super-fluid [53,54] or a Tonks-Girardeau gas [55].

\subsection{GP equation in two and three space variables}

In addition to the programs in one space variable we have six programs in two and three space variables. The programs imagtime2d.F and realtime2d.F apply to 2D Cartesian space using imaginaryand real-time propagations, respectively. Similarly, imagtime3d.F and realtime3d.F apply to 3D Cartesian space using imaginary- and real-time propagation, respectively. Finally, programs imagtimeaxial.F and realtimeaxial.F apply to an axially symmetric trap in 3D. The Fortran 90/95 versions of these programs are somewhat more condensed and provide some advantage. Hence we also include these programs as imagtime2d.f90, realtime2d.f90, imagtime3d.f90, realtime3d.f90, imagtimeaxial.f90, and realtimeaxial.f90. The output of the Fortran 90/95 programs are identical with their corresponding Fortran 77 versions. All these programs are written using a very similar logic used in the programs in one space variable. So most of the considerations described earlier also applies to these cases. We describe the principal differences below.

In case of total number of space points $\mathrm{N}$, one now has the variables NX, NY, and NZ for total number of space points in $\mathrm{X}, \mathrm{Y}$ and $\mathrm{Z}$ directions in case of three space variables. In case of two space variables the $\mathrm{Z}$ component is absent. These variables should be chosen equal to each other for a nearly symmetric case. However, if the problem is anisotropic in space, these variables can and should be chosen differently. Similarly, the space variable $\mathrm{X}(\mathrm{I})$ is now replaced by space variables $\mathrm{X}(\mathrm{I}), \mathrm{Y}(\mathrm{J})$, and $\mathrm{Z}(\mathrm{K})$ in three directions. Now there are space steps DX, DY, and DZ in place of DX in one space variable. The variables DX, DY, and DZ can now be chosen differently in three directions in case of an anisotropic problem. However, they should 
be chosen together with NX, NY, and NZ so that the wave function lies entirely inside the chosen space and becomes negligibly small at the boundaries. If the spatial extension of the wave function is much smaller in one space direction than another, one should take a smaller space step in the direction in which the spatial extension of the wave function is small. The potential $\mathrm{V}$ and wave function $\mathrm{CP}$ are now functions of 2 or 3 space variables and are represented by matrices in place of a column in the case of one space variable. The variables AL (or KAP) and BL (or LAM) now define the anisotropy of the trap and are used in the subroutine INITIALIZE to define the trap. The subroutine LU is now replaced by LUX, LUY, and LUZ to implement the solution in different space directions. In the imaginary-time propagation each time the subroutines LUX, LUY, and LUZ operate, one has to set the normalization of the wave function to unity by calling the subroutine NORM.

\subsection{Instruction to use the programs}

The programs, as supplied, solve the GP equations for a specific value of nonlinearity and write the wave function, chemical potential, energy, rms size or radius, wave function at the center, and nonlinearity, for specific values of space and time steps. The real- and imaginary-time programs for a specific equation, for example, spherically-symmetric 3D equation, employ similar set of parameters like space and time steps. The real-time programs use a larger value of $\mathrm{N}$, so that the discretization covers a larger region in space. In all cases the supplied programs solve an equation of type (4) with a factor of $1 / 2$ in front of the space derivative, selected by setting the integer parameter OPTION $=2$ in the MAIN routine. Other types of equations can be obtained by setting OPTION $=1$ for equations of type (5) or $=3$ for equations of type (2).

For solving a stationary ground state problem, the imaginary-time programs are far more accurate and should be used. The real-time programs should be used for studying non-equilibrium problems often using an initial wave function calculated by the imaginary-time program. The non-equilibrium problems include the study of soliton dynamics [56], expansion [57], collapse dynamics [52], and other types of problems.

Each program is preset at a fixed nonlinearity G0 $(=\mathrm{G})$, correlated DX-DT values and NSTP, NPAS, and NRUN. Smaller the steps DX and DT, more accurate will be the result. The correlated values of DX and DT on a data line should be found by trial to obtain good convergence. Each supplied program produces result up-to a desired precision consistent with the parameters employed - G0, DX, DT, N, NSTP, NPAS, and NRUN. If the nonlinearity G0 is increased, one might need to increase $\mathrm{N}$ to achieve similar precision. In many cases one may need an approximate solution (with lower accuracy) involving less CPU time or one may need to solve the GP equation for a different value of nonlinearity.

(a) If G0 is reduced, just change the card defining G0. However, if G0 is increased, changing the value of G0 may not be enough. For an increased G0, the wave function extends to a larger region in space. One may need to increase the "Number of space mesh points". For a new G0, just plot the output file fort.3 for all programs except realtime3d.F, realtime3d.f90, imagtime3d.F and imagtime3d.f90, where one should plot output files fort.11, fort.12, and fort.13 and see that the wave function is fully and adequately accommodated in the space domain. If not, one needs to increase the number in input cards "Number of space mesh points" until the wave function is fully and adequately accommodated in the space domain.

(b)The CPU time involved can be reduced by sacrificing the precision. This can be done by increasing the space step(s) and time step and reducing the "Number of space mesh points". If the space step DX is increased by a factor of $\mathrm{f}=2$, the number of space mesh points should be reduced by the same factor. The time step DT should be increased by a larger factor, more like $f^{* *} 2$. The optimum increase in time step should be determined by some experimentation. (A set of correlated DX-DT values is given in Tables 1 and 4 for the solution of corresponding equations.) The CPU time is the largest in the case of programs realtime3d.F, realtime3d.f90, imagtime3d.F and imagtime3d.f90 and we give an example of changes below in these cases to reduce the CPU time and precision. For example, for realtime3d.F, just change the lines 15, 16, 17, and 39. The new set of lines should be

PARAMETER $(\mathrm{NX}=40, \mathrm{NXX}=\mathrm{NX}-1, \mathrm{NX} 2=\mathrm{NX} / 2)$

PARAMETER $(\mathrm{NY}=32, \mathrm{NYY}=\mathrm{NY}-1, \mathrm{NY} 2=\mathrm{NY} / 2)$ 
PARAMETER $(\mathrm{NZ}=24, \mathrm{NZZ}=\mathrm{NZ}-1, \mathrm{NZ2}=\mathrm{NZ} / 2)$

DATA DX /0.5D0/, DY /0.5D0/, DZ /0.5D0/, DT/0.05D0/

For imagtime3d.F, just change the lines $15,16,17$, and 37 . The new set of lines should be

PARAMETER $(\mathrm{NX}=24, \mathrm{NXX}=\mathrm{NX}-1, \mathrm{NX} 2=\mathrm{NX} / 2)$

PARAMETER $(\mathrm{NY}=20, \mathrm{NYY}=\mathrm{NY}-1, \mathrm{NY} 2=\mathrm{NY} / 2)$

PARAMETER $(\mathrm{NZ}=16, \mathrm{NZZ}=\mathrm{NZ}-1, \mathrm{NZ2}=\mathrm{NZ} / 2)$

DATA DX /0.5D0/, DY /0.5D0/, DZ /0.5D0/, DT/0.04D0/

Please verify, by running the corresponding programs, that the new results so obtained are quite similar to the existing results.

The integer parameter NSTP refers to the number of time iterations during which the nonlinear term is slowly introduced in the real-time propagation. This number should be large (typically more than 100,000 for small nonlinearity, for larger nonlinearity could be 1000,000 ) for good convergence; this means that the nonlinearity should be introduced in small amounts over a large number of time iterations. In real-time propagation, NPAS refers to certain number of time iterations with the constant nonlinear term already introduced in NSTP and should be small (typically 1000). NRUN refers to time iterations with a modified nonlinearity so as to generate a non-equilibrium dynamics. In the imaginary-time propagation the parameters NPAS and NRUN refer to certain number of time iterations with the constant nonlinear term already introduced in one step and should be large (typically NPAS $=200,000$ or more) for good convergence.

\subsection{Output files}

The programs write via statements $\operatorname{WRITE}\left(1,{ }^{*}\right), \operatorname{WRITE}\left(2,{ }^{*}\right) \operatorname{WRITE}\left(3,{ }^{*}\right)$ in Files 1,2 , and 3 , respectively, the initial stationary wave function and that after NSTP, and NPAS time iterations for real-time propagation and after NPAS and NRUN time iterations for imaginary-time propagation. File 3 gives the final stationary wave function of the calculation. However, in the case of the anisotropic 3D programs realtime3d.F and imagtime3d.F, sections of the wave function as plotted in Fig. 4 (b) are written in Files 1, 2, and 3 before NSTP (realtime3d.F) and after NPAS (imagtime3d.F) iterations, and in Files 11, 12, and 13 after NPAS (realtime3d.F) and NRUN (imagtime3d.F) iterations.

In the real-time program a non-stationary oscillation is initiated by suddenly modifying the nonlinearity from G to G/2 after NPAS time iterations. During NRUN time iterations the non-stationary dynamics is studied. The real-time programs write on File 8 the running time and rms size during non-stationary oscillation.

In addition, these programs write in File 7 the values of nonlinearity G, space steps DX, DY, DZ, time step DT, number of space mesh points N, number of time iterations NSTP, NPAS, and NRUN together with the values of normalization of the wave function, chemical potential, energy, rms size, value of the wave function at center, nonlinearity coefficient $\mathrm{G}$.

Below we provide some sample output listed on File 7 from the different programs using OPTION $=2$. File 7 (fort.7) represents the comprehensive result in each case.

(1) Program imagtime1d.F

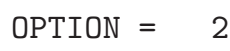

\begin{tabular}{|c|c|c|c|c|c|}
\hline & Norm & Chem & Ener & $\langle x\rangle$ & Psi(0) \\
\hline itial : & 1.0000 & 0.500000 & 0.500000 & 0.70711 & 0.75113 \\
\hline
\end{tabular}




$\begin{array}{lrrrrr}\text { After NPAS iter.: } & 0.9996 & 10.369462 & 6.256976 & 2.04957 & 0.40606 \\ \text { After NRUN iter.: } & 0.9996 & 10.369462 & 6.256976 & 2.04957 & 0.40606\end{array}$

(2) Program imagtimesph.F

OPTION $=2$

\# Space Stp $N=3000$

\# Time Stp : , NPAS $=200000$, NRUN $=20000$

Nonlinearity $\mathrm{G}=\quad 125.48400000$

Space Step DX $=0.002500$, Time Step DT $=0.000020$

\begin{tabular}{|c|c|c|c|c|c|}
\hline & Norm & Chem & Ener & $\langle r\rangle$ & Psi(0) \\
\hline Initial : & 1.0000 & 1.500000 & 1.500000 & 1.22474 & 0.42378 \\
\hline After NPAS iter.: & 0.9998 & 4.014113 & 3.070781 & 1.88214 & 0.17382 \\
\hline After NRUN iter.: & 0.9998 & 4.014113 & 3.070781 & 1.88214 & 0.17382 \\
\hline
\end{tabular}

(3) Program imagtimecir.F

OPTION $=2$

\# Space Stp $N=2000$

\# Time Stp : NPAS $=200000$, NRUN $=200000$

Nonlinearity $\mathrm{G}=\quad-2.50970000$

Space Step DX $=0.002500$, Time Step DT $=0.000020$

\begin{tabular}{|c|c|c|c|c|c|}
\hline & Norm & Chem & Ener & $\langle r\rangle$ & Psi (0) \\
\hline Initial : & 1.0000 & 1.000000 & 1.000000 & 1.00000 & 0.56419 \\
\hline After NPAS iter.: & 1.0000 & 0.499772 & 0.770107 & 0.87758 & 0.67535 \\
\hline After NRUN iter.: & 1.0000 & 0.499772 & 0.770107 & 0.87758 & 0.67535 \\
\hline
\end{tabular}

(4) Program imagtime2d.F and imagtime2d.f90

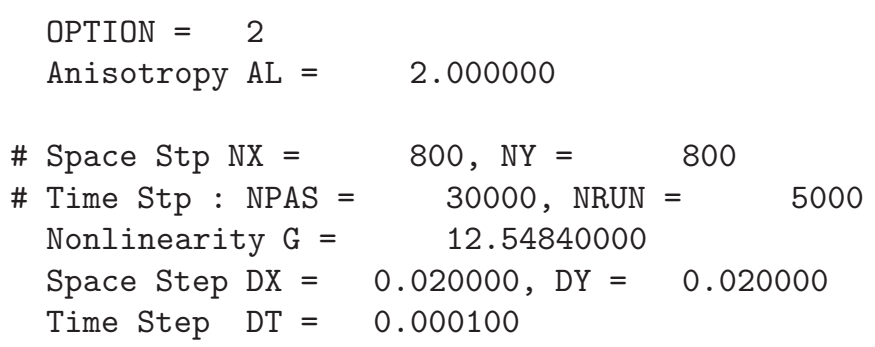

Norm Chem $\quad$ Ener $\quad\langle r>\quad P s i(0,0)$




\begin{tabular}{|c|c|c|c|c|c|}
\hline Initial : & 1.0000 & 1.500000 & 1.500000 & 0.86603 & 0.67094 \\
\hline After NPAS iter.: & 0.9999 & 3.254878 & 2.490493 & 1.17972 & 0.46325 \\
\hline After NRUN iter.: & 0.9999 & 3.254878 & 2.490493 & 1.17972 & 0.46325 \\
\hline
\end{tabular}

(5) Program imagtimeaxial.F and imagtimeaxial.f90 OPTION $=2$

Anisotropy KAP $=1.000000$, LAM $=4.000000$

$\begin{array}{lcc}\text { \# Space Stp NX }= & 500, \text { NY }= & 500 \\ \text { \# Time Stp : NPAS }= & 100000, \text { NRUN }= & 20000\end{array}$

Nonlinearity $\mathrm{G}=18.81000000$

Space Step DX $=0.020000$, DY $=0.020000$

Time Step DT $=0.000040$

\begin{tabular}{|c|c|c|c|c|c|c|c|}
\hline & Norm & Chem & Energy & $\langle$ rho $\rangle$ & $\langle z\rangle$ & $\langle r\rangle$ & psi(0) \\
\hline Initial & $: 1.000$ & 3.00000 & 3.00000 & 1.00000 & 0.35355 & 1.06066 & 0.59883 \\
\hline NPAS iter & $: 1.000$ & 4.36113 & 3.78228 & 1.32490 & 0.38049 & 1.37846 & 0.38129 \\
\hline NRUN iter & $: 1.000$ & 4.36113 & 3.78228 & 1.32490 & 0.38049 & 1.37846 & 0.38129 \\
\hline
\end{tabular}

(6) Program imagtime3d.F and imagtime3d.f90

OPTION $=2$

Anisotropy AL $=1.414214, \mathrm{BL}=2.000000$

\# Space Stp NX $=\quad 240, N Y=200, N Z=160$

\# Time Stp : NPAS $=5000$, NRUN $=500$

$\begin{array}{lcc}\text { Nonlinearity G }= & 44.90700000 & \\ \text { Space Step DX }= & 0.050000, \text { DY }=0.050000, \text { DZ }=0.050000\end{array}$

Time Step DT $=0.000400$

\begin{tabular}{|c|c|c|c|c|c|}
\hline & Norm & Chem & Ener & $\langle r\rangle$ & $\operatorname{Psi}(0,0,0)$ \\
\hline Initial & 1.0000 & 2.2071 & 2.2071 & 1.0505 & 0.5496 \\
\hline After NPAS iter.: & 0.9997 & 4.3446 & 3.4862 & 1.4583 & 0.2888 \\
\hline After NRUN iter.: & 0.9997 & 4.3446 & 3.4862 & 1.4583 & 0.2888 \\
\hline
\end{tabular}

(7) Program realtime1d.F

OPTION $=2$

\# Space Stp N = 5000

\# Time Stp : NSTP 1000000, NPAS $=1000$, NRUN $=40000$

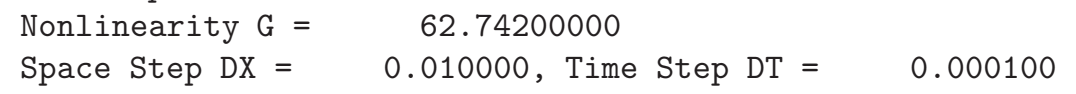

Norm Chem $\quad$ Ener $\quad\langle x\rangle$ Psi $(0)$




\begin{tabular}{|c|c|c|c|c|c|}
\hline \multirow[b]{2}{*}{ Initial : } & \multicolumn{5}{|c|}{-------------} \\
\hline & 1.0000 & 0.500 & 0.500 & 0.707 & 0.751 \\
\hline After NSTP iter.: & 1.0000 & 10.368 & 6.257 & 2.050 & 0.406 \\
\hline After NPAS iter.: & 1.0000 & 10.375 & 6.257 & 2.047 & 0.406 \\
\hline
\end{tabular}

(8) Program realtimesph.F

OPTION $=2$

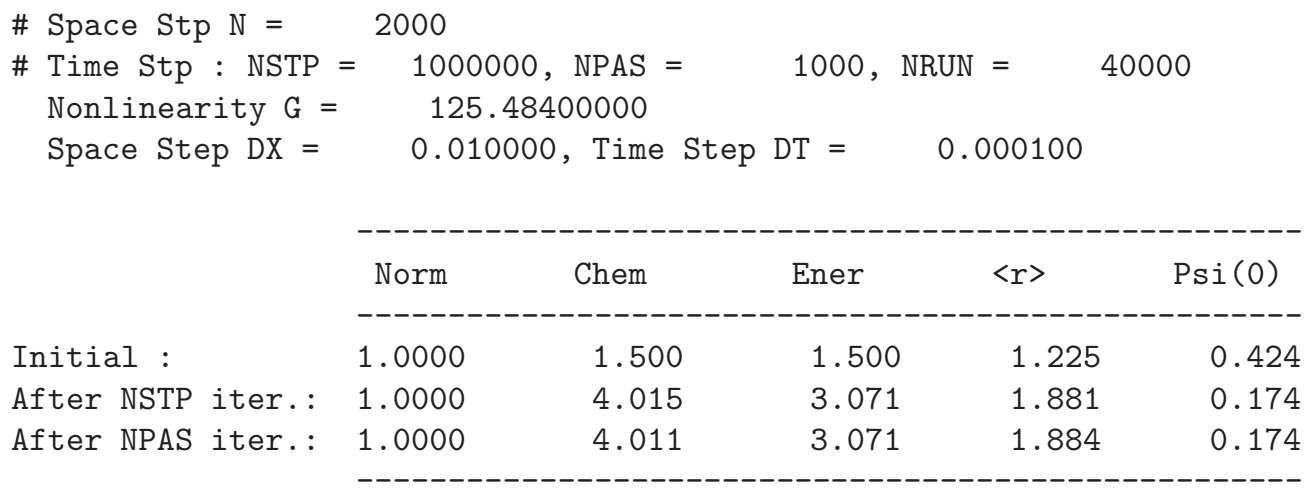

(9) Program realtimecir.F

OPTION $=2$

\# Space Stp $N=2000$

\# Time Stp : NSTP $=1000000$, NPAS $=1000$, NRUN $=40000$

Nonlinearity $\mathrm{G}=\quad 12.54840000$

Space Step DX $=0.010000$, Time Step DT $=0.000100$

\begin{tabular}{|c|c|c|c|c|c|}
\hline & \\
\hline & Norm & Chem & Ener & $\langle r\rangle$ & Psi(0) \\
\hline Initial : & 1.0000 & 1.000 & 1.000 & 1.000 & 0.564 \\
\hline After NSTP iter.: & 1.0000 & 2.255 & 1.708 & 1.308 & 0.391 \\
\hline After NPAS iter.: & 1.0000 & 2.255 & 1.708 & 1.308 & 0.391 \\
\hline
\end{tabular}

(10) Program realtime2d.F and realtime2d.f90

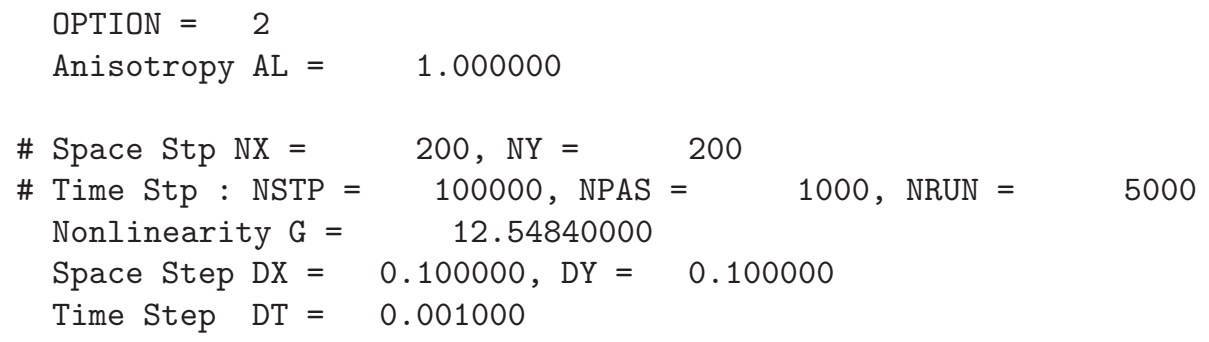

Norm $\quad$ Chem $\quad$ Ener $\quad\langle r\rangle \quad$ Psi $(0,0)$




\begin{tabular}{|c|c|c|c|c|c|}
\hline Initial : & 1.000 & 1.000 & 1.000 & 1.000 & 0.564 \\
\hline After NSTP iter.: & 1.000 & 2.256 & 1.708 & 1.307 & 0.392 \\
\hline After NPAS iter.: & 1.000 & 2.257 & 1.708 & 1.305 & 0.392 \\
\hline
\end{tabular}

(11) Program realtimeaxial.F and realtimeaxial.f90

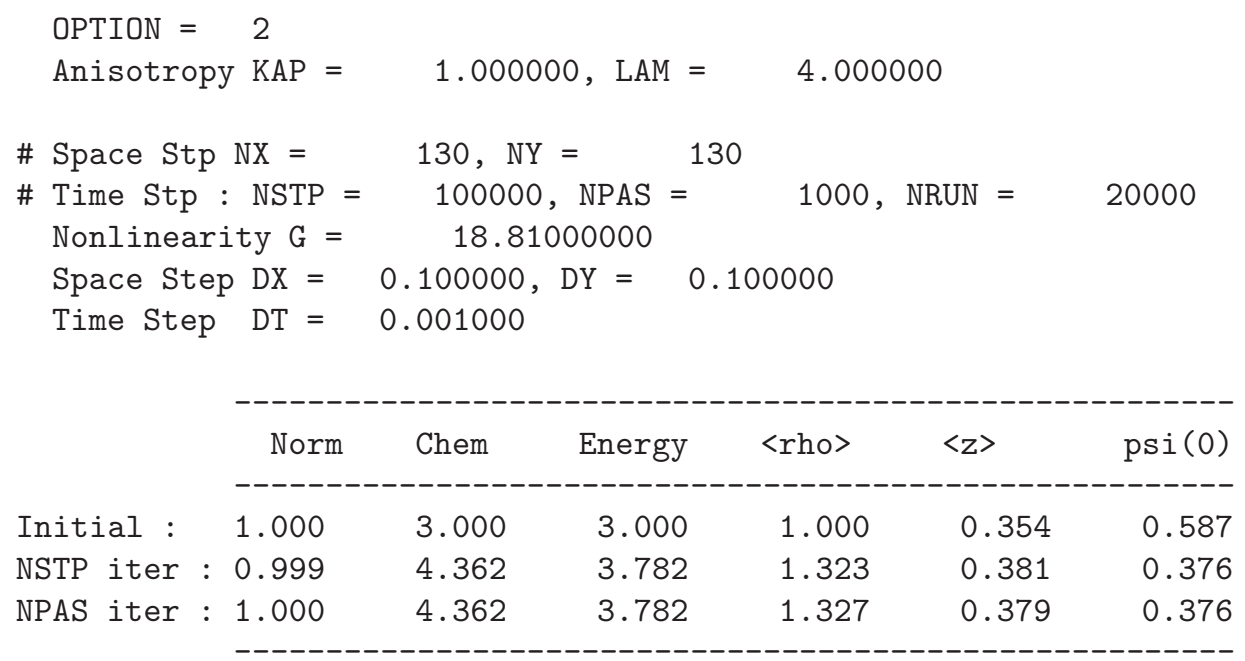

(12) Program realtime3d.F and realtime3d.f90

OPTION $=2$

Anisotropy $\mathrm{AL}=1.414214, \mathrm{BL}=2.000000$

\# Space Stp NX $=\quad 200, \mathrm{NY}=160, \mathrm{NZ}=120$

\# Time Stp : NSTP $=60000$, NPAS $=4000$

$\begin{array}{ll}\text { Nonlinearity G }= & 22.45400000 \\ \text { Space Step DX }= & 0.100000, \text { DY }=0.100000, \text { DZ }=0.100000\end{array}$

Time Step DT $=0.002000$

\begin{tabular}{|c|c|c|c|c|c|}
\hline & Norm & Chem & Ener & $\langle r\rangle$ & $\operatorname{Psi}(0,0,0)$ \\
\hline Initial : & 1.000 & 2.207 & 2.207 & 1.051 & 0.550 \\
\hline After NSTP iter.: & 1.000 & 3.572 & 2.992 & 1.321 & 0.347 \\
\hline After NPAS iter.: & 1.000 & 3.572 & 2.992 & 1.320 & 0.347 \\
\hline
\end{tabular}




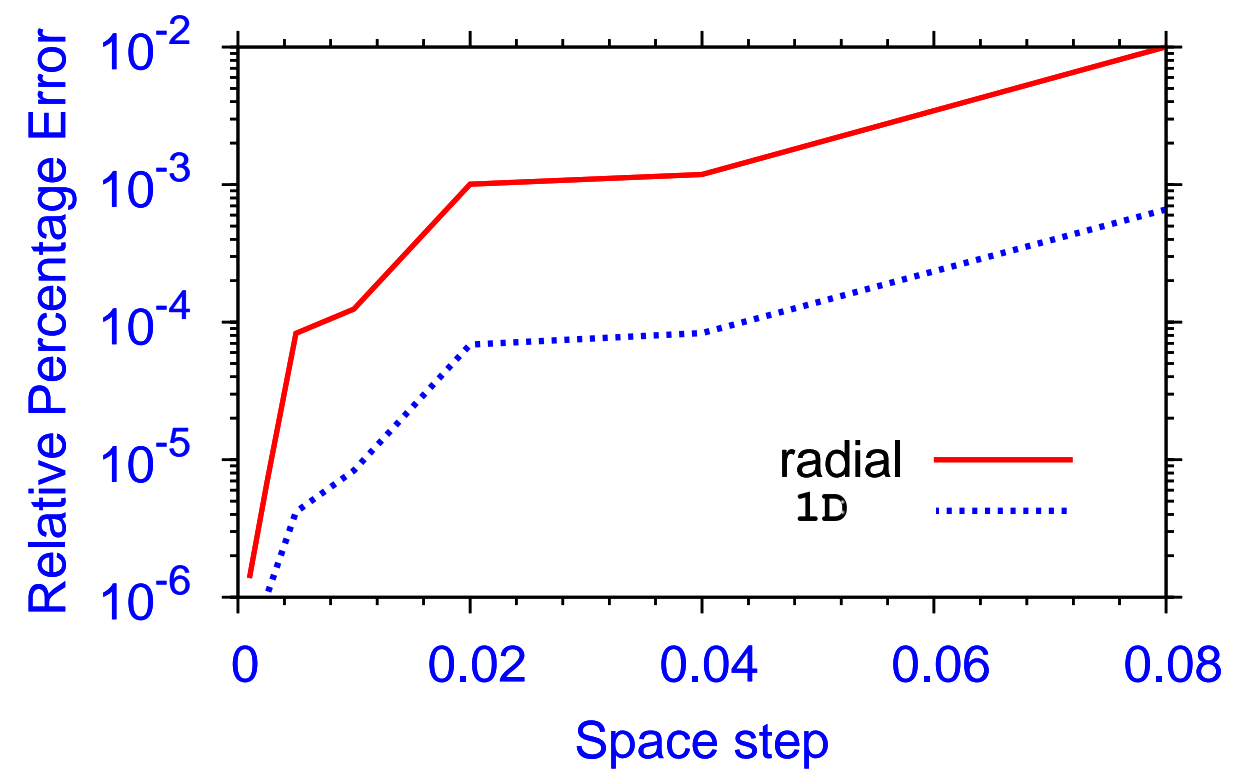

Fig. 1. (Color online) Relative percentage error as a function of space step for one-dimensional (1D) and spherically-symmetric 3D (radial) models with nonlinearity $\aleph=627.42$ and 627.4 , respectively, calculated using the imaginary-time propagation programs imagtime1d.F [Eq. (15), OPTION 2] and imagtimesph.F [Eq. (4), OPTION 2] with the data of Table 1.

\section{Numerical Results}

\subsection{Stationary Problem}

Table 1

Convergence of result in the 1D (X) and radially-symmetric 3D $(r)$ cases for nonlinearities $\aleph=627.42$ and 627.4 , calculated using the imaginary-time propagation programs imagtime1d.F [Eq. (15), OPTION 2] and imagtimesph.F [Eq. (4), OPTION 2] respectively, for various space step DX and time step DT.

\begin{tabular}{|r|r|r|r|r|r|}
\hline$\aleph$ & $\mathrm{DX}$ & $\mathrm{DT}$ & $\varphi(0) / \phi(0)$ & $x_{\mathrm{rms}} / r_{\mathrm{rms}}$ & $\mu$ \\
\hline $627.42(\mathrm{X})$ & 0.08 & 0.005 & 0.276647 & 4.384825 & 48.024062 \\
$627.42(\mathrm{X})$ & 0.04 & 0.001 & 0.276648 & 4.384744 & 48.024389 \\
$627.42(\mathrm{X})$ & 0.02 & 0.0005 & 0.276649 & 4.384734 & 48.024429 \\
$627.42(\mathrm{X})$ & 0.01 & 0.0001 & 0.276649 & 4.384726 & 48.024462 \\
$627.42(\mathrm{X})$ & 0.005 & 0.00005 & 0.276649 & 4.384725 & 48.024466 \\
$627.42(\mathrm{X})$ & 0.0025 & 0.00002 & 0.276649 & 4.384724 & 48.024468 \\
$627.42(\mathrm{X})$ & 0.001 & 0.00001 & 0.276649 & 4.384724 & 48.024468 \\
\hline $627.4(r)$ & 0.08 & 0.005 & 0.106655 & 2.506348 & 7.247479 \\
$627.4(r)$ & 0.04 & 0.001 & 0.106679 & 2.505886 & 7.248206 \\
$627.4(r)$ & 0.02 & 0.0005 & 0.106684 & 2.505833 & 7.248292 \\
$627.4(r)$ & 0.01 & 0.0001 & 0.106686 & 2.505785 & 7.248365 \\
$627.4(r)$ & 0.005 & 0.00005 & 0.106686 & 2.505780 & 7.248374 \\
$627.4(r)$ & 0.0025 & 0.00002 & 0.106686 & 2.505776 & 7.248380 \\
$627.4(r)$ & 0.001 & 0.00001 & 0.106686 & 2.505776 & 7.248380 \\
\hline
\end{tabular}

In this subsection we present results for the stationary ground state problem calculated with the imaginarytime programs. All numerical results presented in this paper are for the nonlinear equations with a factor 
Table 2

The chemical potential $\mu$, rms radius $r_{\mathrm{rms}}$, and the wave function $\phi(0)$ at the center for various nonlinearities in the radially symmetric 3D case calculated using the program imagtimesph.F [Eq. (4), OPTION 2]. Table completed with space step DR $\leq 0.0025$ and $\mathrm{DT}=0.00002$.

\begin{tabular}{|r|r|r|r|r|r|r|}
\hline$\aleph$ & $\phi(0)$ & $\phi(0)[4]$ & $r_{\mathrm{rms}}$ & $r_{\mathrm{rms}}[4]$ & $\mu$ & $\mu[3,4]$ \\
\hline-3.1371 & $0.48792(1)$ & 0.4881 & $1.51213(1)$ & 1.1521 & $1.265184(2)$ & 1.2652 \\
0 & 0.42378 & 0.4238 & 1.22474 & 1.2248 & 1.500000 & 1.5000 \\
3.1371 & $0.38425(1)$ & 0.3843 & $1.27857(1)$ & 1.2785 & $1.677451(1)$ & 1.6774 \\
12.5484 & $0.31800(1)$ & 0.3180 & $1.39211(1)$ & 1.3921 & $2.065018(1)$ & 2.0650 \\
31.371 & $0.25810(1)$ & 0.2581 & $1.53561(1)$ & 1.5356 & $2.586116(1)$ & 2.5861 \\
125.484 & $0.17382(1)$ & 0.1738 & $1.88215(1)$ & 1.8821 & $4.014113(2)$ & 4.0141 \\
627.4 & $0.10669(1)$ & 0.1066 & $2.50578(1)$ & 2.5057 & $7.248380(3)$ & 7.2484 \\
3137.1 & $0.06559(1)$ & 0.0655 & $3.41450(1)$ & 3.4145 & $13.553403(4)$ & 13.553 \\
\hline
\end{tabular}

of $1 / 2$ in front of the gradient term obtained by choosing OPTION $=2$ in the MAIN. First we consider the numerical result for the simplest cases - the 1D and the radially-symmetric 3D problems by imaginary-time propagation with nonlinearities $\aleph=627.42$ and 627.4 , respectively. The calculations were performed with different space and time steps DX and DT, respectively. (As DX is reduced, DT should be reduced also to have good convergence. The correlated DX-DT values were obtained by trial to achieve good convergence.) In each case a sufficiently large number of space points $\mathrm{N}$ is to be taken, so that the space domain of integration covers the extension of the wave function adequately. We exhibit in Table 1, the results for the wave function at center, rms size, and chemical potential in these two cases for a fixed nonlinearity for different DX and DT. We find that convergence is achieved up to six significant digits after the decimal point with space step DX $=0.0025$ and time step DT $=0.00002$. In Fig. 1 we plot the relative percentage error in chemical potential $\mu$ for various space steps. The percentage error rapidly reduces as space step is reduced.

In Table 2 we exhibit the chemical potential, rms radius, and the wave function at the center for various nonlinearities in the spherically symmetric 3D case using space step DX $=0.0025$ and time step DT $=0.00002$ in the imaginary-time propagation program. From the table we find that the results are in agreement with those calculated in Refs. [3,4].

Table 3

The chemical potential $\mu$, rms size $x_{\mathrm{rms}}$, and the wave function $\varphi(0)$ at the center for various nonlinearities in the 1D case calculated using the program imagtime1d.F [Eq. (15), OPTION 2]. Table completed with DX $\leq 0.0025$ and DT $=0.00002$.

\begin{tabular}{|r|r|r|r|r|r|r|}
\hline$\aleph$ & $\varphi(0)$ & $\varphi(0)[4]$ & $x_{\mathrm{rms}}$ & $x_{\mathrm{rms}}[4]$ & $\mu$ & $\mu[4]$ \\
\hline-2.5097 & $0.91317(1)$ & 0.9132 & $0.51334(1)$ & 0.5133 & $-0.80623(3)$ & -0.8061 \\
0 & 0.75112 & 0.7511 & 0.70711 & 0.7071 & 0.500000 & 0.5000 \\
3.1371 & $0.64596(1)$ & 0.6459 & $0.89602(1)$ & 0.8960 & $1.526593(3)$ & 1.5265 \\
12.5484 & $0.52975(1)$ & 0.5297 & $1.24549(1)$ & 1.2454 & $3.596560(2)$ & 3.5965 \\
31.371 & $0.45567(1)$ & 0.4556 & $1.64170(1)$ & 1.6416 & $6.552682(2)$ & 6.5526 \\
62.742 & $0.40606(1)$ & 0.4060 & $2.04957(1)$ & 2.0495 & $10.369462(2)$ & 10.369 \\
156.855 & $0.34856(1)$ & 0.3485 & $2.76794(1)$ & 2.7679 & $19.070457(2)$ & 19.0704 \\
313.71 & $0.31053(1)$ & 0.3105 & $3.48237(1)$ & 3.4823 & $30.259178(3)$ & 30.259 \\
627.42 & $0.27665(1)$ & 0.2766 & $4.38472(1)$ & 4.3847 & $48.024468(3)$ & 48.024 \\
1254.8 & $0.24647(1)$ & 0.2464 & $5.52282(1)$ & 5.5228 & $76.226427(3)$ & 76.226 \\
\hline
\end{tabular}

In Table 3 we exhibit the chemical potential, rms size, and the wave function at the center for various nonlinearities in the $1 \mathrm{D}$ case using space step DX $=0.0025$ and time step DT $=0.00002$. From the table we find that the results are in agreement with those calculated in Ref. [4]. 

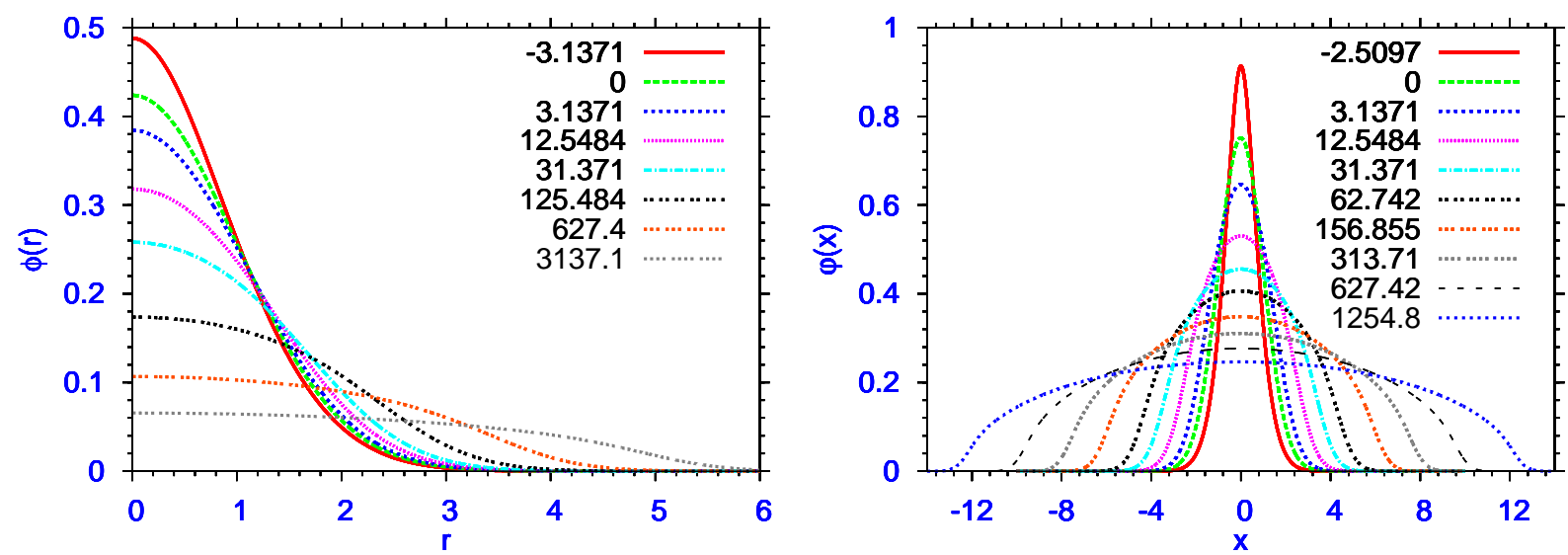

Fig. 2. (Color online) Plot of wave function profile for the (a) radially-symmetric 3D case [Eq. (4), using imagtimesph.F, OPTION 2] and (b) 1D case [Eq. (15), using imagtime1d.F, OPTION 2]. The curves are labeled by their respective nonlinearities as tabulated in Tables 2 and 3, respectively.

Table 4

Convergence of results for chemical potential $\mu$, rms size $r_{\text {rms }}$ and the wave function $\phi(0)$ at the center in the Cartesian $2 \mathrm{D}$ case for a nonlinearity $\aleph=12.5484$ and anisotropy $\kappa=1$ for different space steps $h \equiv \mathrm{DX}=\mathrm{DY}$ obtained from the program imagtime2d.F [using Eq. (19), OPTION 2].

\begin{tabular}{|r|r|r|r|r|r|}
\hline$\aleph$ & $\mathrm{DX}=\mathrm{DY}$ & $\mathrm{DT}$ & $\varphi(0)$ & $r_{\mathrm{rms}}$ & $\mu$ \\
\hline 12.5484 & 0.10 & 0.01 & $0.39189(2)$ & $1.30678(3)$ & 2.2559 \\
12.5484 & 0.08 & 0.005 & $0.39190(2)$ & $1.30685(3)$ & 2.2559 \\
12.5484 & 0.06 & 0.003 & $0.39190(2)$ & $1.30690(3)$ & $2.25582(3)$ \\
12.5484 & 0.04 & 0.001 & $0.39190(2)$ & $1.30693(2)$ & $2.25579(2)$ \\
12.5484 & 0.02 & 0.0005 & $0.39190(2)$ & $1.30687(2)$ & $2.25583(2)$ \\
12.5484 & 0.015 & 0.0003 & $0.39190(2)$ & $1.30687(2)$ & $2.25583(2)$ \\
12.5484 & 0.01 & 0.0001 & $0.39190(2)$ & $1.30687(2)$ & $2.25583(2)$ \\
\hline
\end{tabular}

In Figs. 2 (a) and (b) we plot the wave function profiles for the radially-symmetric 3D and 1D cases calculated using the programs imagtimesph.F and imagtimeld.F, respectively, for different nonlinearities presented in Tables 2 and 3. As the nonlinearity is increased the system becomes more repulsive and the wave function extends to a larger domain in space.

In Table 4 we present the results for the wave function at center, rms radius, and chemical potential for the Cartesian 2D case with nonlinearity $\aleph=12.5484$ using the imaginary-time propagation program imagtime2d.F. We find that desired convergence is achieved with space step DX $=0.02$. (Note that the converged result in this case is less accurate than those in Tables 1, 2 and 3 as we have used a larger space step in order to keep the CPU time small. A finer mesh will increase the accuracy requiring a larger CPU time.)

In Table 5 we present results for the wave function at the center, rms size, and chemical potential for different nonlinearities in the anisotropic and circularly-symmetric 2D case calculated using the imaginarytime routine imagtime2d.F and imagtimecir.F, respectively. In the anisotropic case we used space step 0.02 and time step 0.0001 and in the circularly-symmetric case we used space step 0.0025 and time step 0.00002 . We also compare these results with those of Ref. [4] and establish more accurate results in the present numerical calculation.

In Figs. 3 (a) and (b) we plot the wave function profiles for the circularly-symmetric and anisotropic $2 \mathrm{D}$ cases using the programs imagtimecir.F and imagtime2D.F, respectively. In the anisotropic 2D case the anisotropy $\kappa=2$ and nonlinearity $\aleph=12.4584$. Because of anisotropy the wave function in Fig. 3 (b) is compressed in the $y$ direction (note the different scales in $x$ and $y$ directions of the plot.) 
Table 5

The chemical potential $\mu$, rms size $r_{\mathrm{rms}}$, and the wave function $\varphi(0)$ at the center for various nonlinearities in the anisotropic 2D case obtained using the programs imagtime2d.F [Eq. (19), OPTION 2] and imagtimecir.F [Eq. (22), OPTION 2]. The case $\kappa=1$ represents circular symmetry and $\kappa \neq 1$ corresponds to anisotropy. Table completed with DX $=$ DY $\leq 0.02$ and DT $=0.0001$ for imagtime2d.F and space step DR $\leq 0.0025$ and DT $=0.00002$ for imagtimecir.F.

\begin{tabular}{|r|r|r|r|r|r|r|r|r|r|r|}
\hline$\kappa$ & $\aleph$ & \multicolumn{3}{|c|}{$\varphi(0)$} & \multicolumn{3}{|c|}{$r_{\mathrm{rms}}$} & & \multicolumn{3}{|c|}{$\mu$} \\
\hline & & anisotropic & circular & {$[4]$} & anisotropic & circular & {$[4]$} & anisotropic & circular & {$[4]$} \\
\hline 1 & -2.5097 & 0.6754 & $0.67532(3)$ & 0.6754 & $0.87759(2)$ & $0.87758(1)$ & 0.8775 & $0.49978(3)$ & $0.49978(1)$ & 0.4997 \\
1 & 0 & 0.5642 & $0.56419(1)$ & 0.5642 & 1.00000 & 1.00000 & 1.0000 & 1.00000 & 1.000000 & 1.0000 \\
1 & 3.1371 & 0.4913 & $0.49128(1)$ & 0.4913 & $1.10513(2)$ & $1.10515(1)$ & 1.1051 & $1.42005(1)$ & $1.420054(3)$ & 1.4200 \\
1 & 12.5484 & 0.3919 & $0.39190(2)$ & 0.3919 & $1.30687(2)$ & $1.30686(1)$ & 1.3068 & $2.25583(1)$ & $2.255840(3)$ & 2.2558 \\
$\sqrt{2}$ & 12.5484 & 0.4267 & & & $1.22054(2)$ & & & $2.69607(1)$ & & \\
2 & 12.5484 & $0.4633(1)$ & & & & & & & \\
1 & 62.742 & 0.2676 & $0.26760(3)$ & 0.2676 & $1.78817(2)$ & $1.78816(1)$ & 1.7881 & $4.60982(1)$ & $4.609831(3)$ & 4.6098 \\
$1 / \sqrt{2}$ & 62.742 & 0.2453 & & & $1.99987(2)$ & & & $3.88210(2)$ & & \\
$1 / 2$ & 62.742 & 0.2249 & & & $2.34157(2)$ & & & $3.27923(2)$ & & \\
1 & 313.71 & 0.1787 & $0.17872(3)$ & 0.1787 & $2.60441(2)$ & $2.60441(1)$ & 2.6044 & $10.06825(3)$ & $10.068262(5)$ & 10.068 \\
1 & 627.42 & 0.1502 & $0.15024(3)$ & 0.1502 & $3.08453(2)$ & $3.08453(2)$ & 3.0845 & $14.18922(3)$ & $14.189228(5)$ & 14.1892 \\
\hline
\end{tabular}
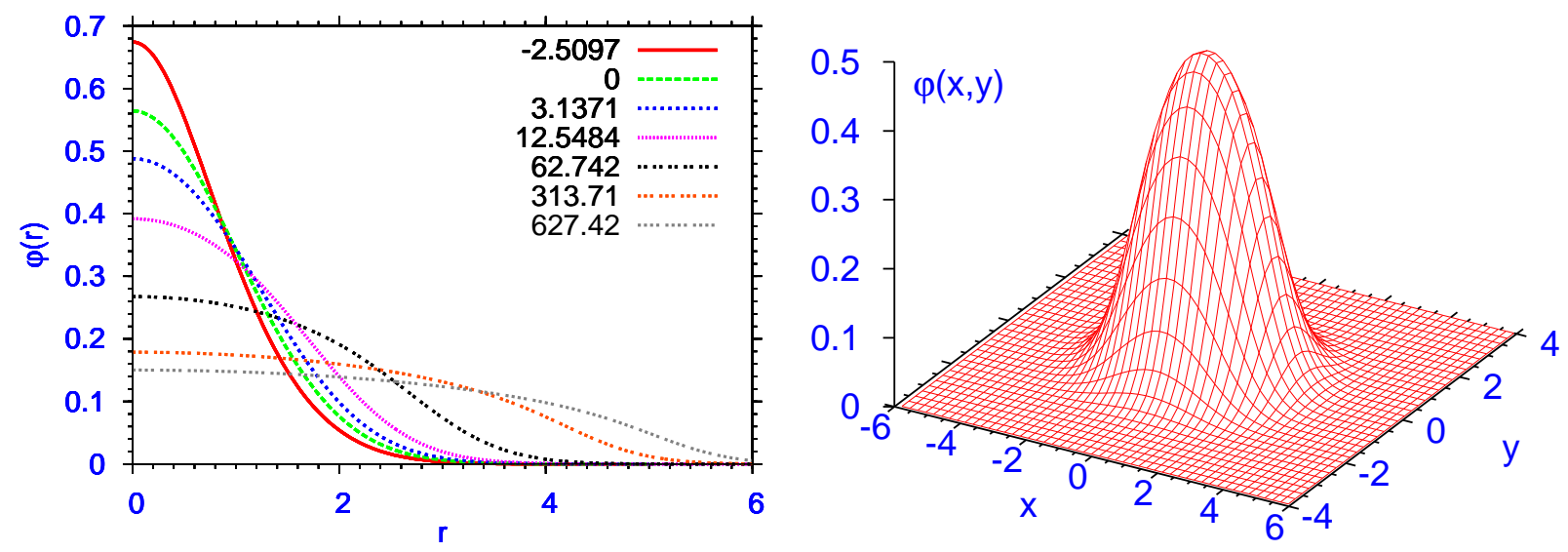

Fig. 3. (Color online) Plot of wave function profile for the (a) circularly-symmetric [Eq. (22), OPTION 2] and (b) anisotropic $2 \mathrm{D}$ cases [Eq. (19), OPTION 2] with nonlinearity $\aleph=12.5484$ and anisotropy $\kappa=2$ using programs imagtimecir.F and imagtime2d.F, respectively. Curves in (a) are labeled by the respective nonlinearities as in Table 5 .

In Table 6 we present results for the wave function at the center, rms sizes, and chemical potential for different nonlinearities in the axially-symmetric 3D case with $\kappa=1$ and $\lambda=4$ calculated using the imaginary-time routine imagtimeaxial.F and space step $D \rho=D Z=0.02$ and time step $D T=0.0004$. We compare with the results of Ref. [4] and establish more accurate results.

In Figs. 4 (a) and (b) we plot the wave function profiles for the axially-symmetric and fully anisotropic $3 \mathrm{D}$ cases using the programs imagtimeaxial.F and imagtime3d.F, respectively. In the axially-symmetric 3D case the anisotropy $\kappa=1, \lambda=4$ and nonlinearity $\aleph=1881$ were employed. In the fully anisotropic 3D case the anisotropies $\nu=1, \kappa=\sqrt{2}$, and $\lambda=2$ and nonlinearities $\aleph=22.454,359.26$ and 11496.3 are used. The effect of the anisotropy is explicit in the $3 \mathrm{D}$ case in generating different profiles of the wave function along $x, y$, and $z$ directions for a fixed nonlinearity.

Next we consider the fully anisotropic case in three dimensions and compare our results with those of Ref. [5] calculated using the program imagtime3d.F. This case mimics a realistic case of experimental interest with $\mathrm{Na}$ atoms. The completely anisotropic trap considered here is time-orbiting potential (TOP) trap with 
Table 6

The chemical potential $\mu$, rms sizes, and the wave function $\varphi(0)$ at the center for various nonlinearities in the axially-symmetric 3D case for ${ }^{87} \mathrm{Rb}$ atoms in an axial trap with $\lambda=4, \kappa=1$ obtained using the program imagtimeaxial.F [Eq. (11), OPTION 2]. The axial frequency is taken as $\omega_{z}=80 \pi \mathrm{Hz}, m\left({ }^{87} \mathrm{Rb}\right)=1.44 \times 10^{-25} \mathrm{~kg}, l=\sqrt{\hbar / m \omega_{x}}=0.3407 \times 10^{-5} \mathrm{~m}, a=5.1 \mathrm{~nm}$, and the ratio between scattering and oscillator length $4 \pi a / l=0.01881$. Table completed with $\mathrm{DZ} \leq 0.02, \mathrm{D} \rho \leq 0.02$ and and DT $=0.00004$.

\begin{tabular}{|r|r|r|r|r|r|r|r|r|r|}
\hline$N$ & $\aleph$ & $\varphi(0)$ & $\varphi(0)[4]$ & $\rho_{\mathrm{rms}}$ & $\rho_{\mathrm{rms}}[4]$ & $z_{\mathrm{rms}}$ & $z_{\mathrm{rms}}[4]$ & $\mu$ & $\mu[4]$ \\
\hline 0 & 0 & $0.5993(1)$ & 0.602 & 1.0000 & 1.000 & 0.3536 & 0.3539 & 3.0000 & 3.0000 \\
1000 & 18.81 & $0.3813(2)$ & 0.3824 & 1.3249 & 1.325 & 0.3805 & 0.3807 & 4.3611 & 4.362 \\
5000 & 94.05 & 0.2474 & 0.2477 & 1.7742 & 1.7742 & 0.4212 & 0.4214 & 6.6797 & 6.680 \\
10000 & 188.1 & 0.2021 & 0.2023 & 2.0411 & 2.041 & 0.4496 & 0.4497 & 8.3671 & 8.367 \\
50000 & 940.5 & 0.1247 & 0.1248 & 2.8424 & 2.842 & 0.5531 & 0.5532 & 14.9487 & 14.95 \\
100000 & 1881 & 0.1011 & 0.1012 & 3.2758 & 3.276 & 0.6173 & 0.6174 & 19.4751 & 19.47 \\
400000 & 7524 & 0.0666 & 0.0666 & 4.3408 & 4.341 & 0.7881 & 0.7881 & 33.4677 & 33.47 \\
800000 & 15048 & 0.0540 & 0.0540 & 4.9922 & 4.992 & 0.8976 & 0.8976 & 44.0234 & 43.80 \\
\hline
\end{tabular}
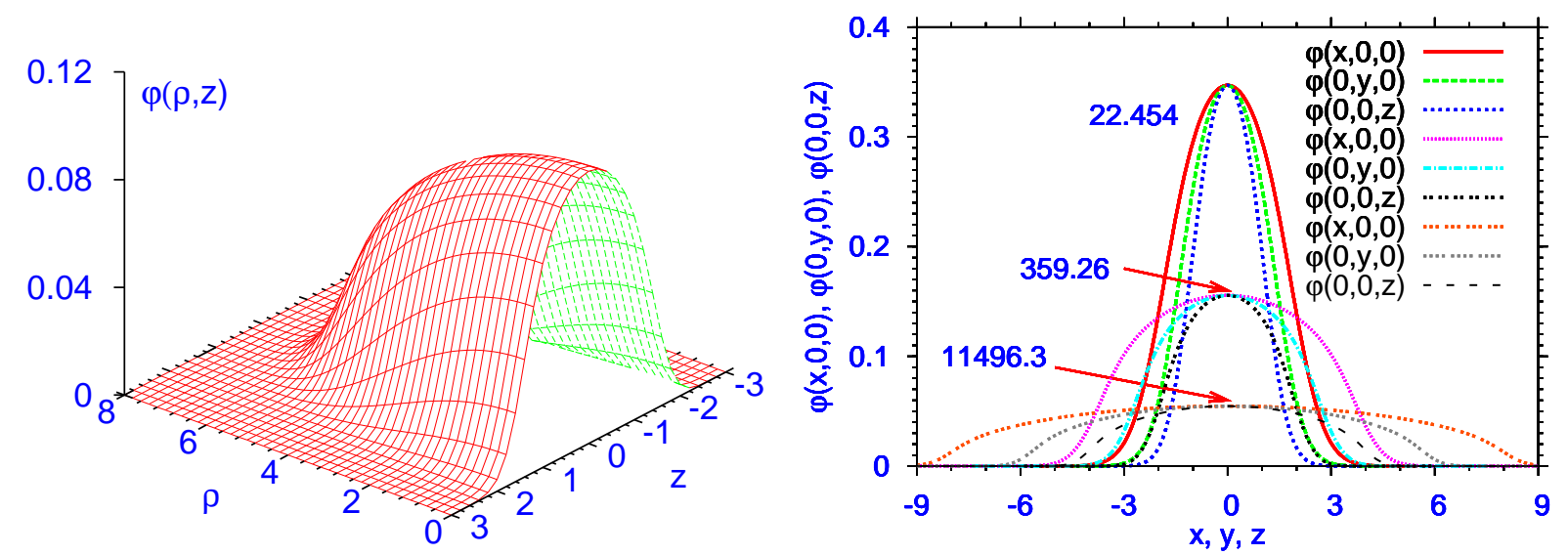

Fig. 4. (Color online) Plot of wave function profile for the (a) axially-symmetric 3D case with nonlinearity $\aleph=1881$ anisotropy $\lambda=4, \kappa=1$ using imagtimeaxial.F [Eq. (11), OPTION 2] and (b) anisotropic 3D case with nonlinearity $\aleph=22.454,359.26$, and 11496.3 and anisotropy $\nu=1, \lambda=\sqrt{2}$ and $\kappa=2$ using imagtime3d.F [Eq. (8), OPTION 2]. In the 3D case only the sections $\varphi(x, 0,0), \varphi(0, y, 0)$ and $\varphi(0,0, z)$ of the wave functions are plotted.

angular frequencies in the natural ratio $\left(\omega_{x}, \omega_{y}, \omega_{z}\right)=\omega_{0}^{A}(1, \sqrt{2}, 2)$, with $\omega_{0}^{A}=354 \pi \mathrm{rad} / \mathrm{s}$. BEC in such a system has been observed by Kozuma et al. [58]. We also consider the spherical potential with $\omega_{0}^{S}=87$ $\mathrm{rad} / \mathrm{s}$ [59]. The s-wave scattering length of $\mathrm{Na}$ is taken as $a=52 a_{0} \approx 2.75 \mathrm{~nm}$, with $a_{0}=0.5292 \AA$ the Bohr radius [5].

In Table 7 we exhibit the results for our calculations with the fully anisotropic potential together with those for the spherically-symmetric potential in three dimensions. The results for the spherically-symmetric potential are also calculated using the one-dimensional radially symmetric imaginary-time program imagtimesph.F in addition to the fully anisotropic program imagtime3d.F. In the anisotropic case the present results are consistent with those of Ref. [5]. In the spherical case the two sets of the present results (calculated with the spherically-symmetric and anisotropic programs) as well as those of Ref. [5] are consistent with each other.

The input to our calculation is the nonlinearity coefficient $\aleph$, which is related to the scattering length $a$, number of atoms $N$ and harmonic oscillator length $l$ via $\aleph=4 \pi a N / l$ in Eq. (8). We provide the nonlinearity values of our calculations. Although the present results are in agreement with those of Ref. [5], a very precise comparison of the two calculations is not to the point as Schneider and Feder did not provide the nonlinearity coefficient $\aleph$ used in their calculation. 
Table 7

The chemical potential $\mu, \mathrm{rms}$ sizes, and wave function $\varphi(0)$ at the center for various number $N$ of condensate of Na atoms. The constants used are $m(\mathrm{Na})=38.175 \times 10^{-27} \mathrm{~kg}, a(\mathrm{Na})=2.75 \mathrm{~nm}$. In all cases the input to numerical calculation was the nonlinearity coefficient $\aleph=4 \pi N a / l$ shown below. For the spherically-symmetric case, we solved the radially symmetric program imagtimesph.F [Eq. (4), OPTION 2], (in addition to the 3D anisotropic program imagtime3d.F setting equal frequencies in all three directions with $\mathrm{DX}=\mathrm{DY}=\mathrm{DZ}=0.05$ and $\mathrm{DT}=0.0004)$ using $[5,59] \omega_{0}^{S}=87 \mathrm{rad} / \mathrm{s}, l=\sqrt{\hbar / m \omega_{0}^{S}}=5.635 \mu \mathrm{m}, \mathrm{DR}$ $\leq 0.0025$ and $\mathrm{DT}=0.00002$. For the fully anisotropic case we used the program imagtime3d.F [Eq. (8), OPTION 2] with [5,58] $\omega_{x} \equiv \omega_{0}^{A}=354 \pi \mathrm{rad} / \mathrm{s}, \omega_{y}=\sqrt{2} \omega_{x}, \omega_{z}=2 \omega_{x}, l=\sqrt{\hbar / m \omega_{0}^{A}}=1.576 \mu \mathrm{m}, \mathrm{DX}=\mathrm{DY}=\mathrm{DZ}=0.05$ and DT $=0.0004$.

\begin{tabular}{|r|r|r|r|r|r|r|r|r|r|}
\hline & \multicolumn{5}{|c|}{ Spherical } & \multicolumn{5}{|c|}{ anisotropic } \\
\hline$N$ & $\aleph$ & $\mu(\mathrm{sph})$ & $\mu(\mathrm{ani})$ & $\mu[5]$ & $\aleph$ & $r_{\mathrm{rms}}$ & $\varphi(0)$ & $\mu($ ani $)$ & $\mu[5]$ \\
\hline 0 & 0 & 1.500000 & 1.5000 & 1.500 & 0 & 1.0505 & 0.5496 & 2.2071 & 2.207 \\
1024 & 6.2798 & $1.824546(1)$ & 1.8245 & 1.825 & 22.454 & 1.3211 & 0.3471 & 3.5718 & 3.572 \\
2048 & 12.5597 & $2.065406(1)$ & 2.0654 & 2.065 & 44.907 & 1.4584 & 0.2888 & 4.3446 & 4.345 \\
4096 & 25.1194 & $2.434526(1)$ & 2.4345 & 2.435 & 89.81 & 1.6328 & 0.2363 & 5.4253 & 5.425 \\
8192 & 50.239 & $2.970180(1)$ & 2.9702 & 2.970 & 179.63 & 1.8460 & 0.1919 & 6.9042 & 6.904 \\
16384 & 100.477 & $3.719211(1)$ & 3.7192 & 3.719 & 359.26 & 2.0999 & 0.1555 & 8.9003 & 8.900 \\
32768 & 200.955 & $4.743445(2)$ & 4.7434 & 4.743 & 718.52 & 2.3979 & 0.1260 & 11.5718 & 11.572 \\
65536 & 401.91 & $6.123751(2)$ & 6.1238 & 6.124 & 1437.03 & 2.7447 & 0.1022 & 15.1284 & 15.128 \\
131072 & 803.82 & $7.970154(2)$ & 7.9702 & 7.970 & 2874.06 & 3.1460 & 0.0829 & 19.8475 & 19.847 \\
262144 & 1607.64 & $10.426912(3)$ & 10.4269 & 10.427 & 5748.13 & 3.6092 & 0.0673 & 26.0961 & 26.096 \\
524288 & 3215.28 & $13.685486(3)$ & 13.6855 & 13.685 & 11496.3 & 4.1426 & 0.0546 & 34.3590 & 34.358 \\
\hline
\end{tabular}

\subsection{Non-stationary Oscillation}

The real-time propagation programs calculate the stationary states under different trap symmetries. However, they are less efficient than the imaginary-time propagation programs in this task requiring more CPU time and producing less accurate results. However, unlike the imaginary-time propagation programs, the real-time programs can produce time evolution of non-stationary states also and next we present results of such time evolution using the real-time propagation programs under different trap symmetries.

In this subsection we present results for non-stationary oscillation obtained with the use of the real-time programs. After the calculation of the stationary profile, the nonlinearity is suddenly reduced to half. The wave function is no longer an eigenstate of the new nonlinear equation. This sets the system into nonstationary oscillation which continues for ever. In Fig. 5 we plot the rms size of the wave function vs. time $t$ showing this oscillation using the output from File 8 for (a) 1D case (using program realtime1d.F), (b) radially-symmetric 3D case (using program realtimesph.F), (c) Cartesian 2D case with anisotropy $\kappa=1$ (using program realtime2d.F), and (d) 3D axially-symmetric case (using program realtimeaxial.F) with respective nonlinearities $\aleph=62.742,125.484,12.5484$, and 18.81 . The rms size at $t=0$ is the rms size of the stationary wave function obtained after NPAS time iterations.

Because of transverse instability, the real-time program realtime3d.F in 3D does not lead to stable sinusoidal oscillation as in other cases for a large change in nonlinearity (nonlinearity reduced to half of its initial value) as shown in Fig. 5. Only for small perturbation a sinusoidal oscillation is observed. However, we do not present a systematic study of such oscillation.

\section{Summary and Conclusion}

In this paper we describe a split-step method for the numerical solution of the time-dependent nonlinear GP equation under the action of a general anisotropic 3D trap using real- and imaginary-time propagation. Similar methods for 1D and anisotropic 2D traps are also described. The time propagation is carried with an initial input. The full Hamiltonian is split into several spatial derivative and a non-derivative parts. The 

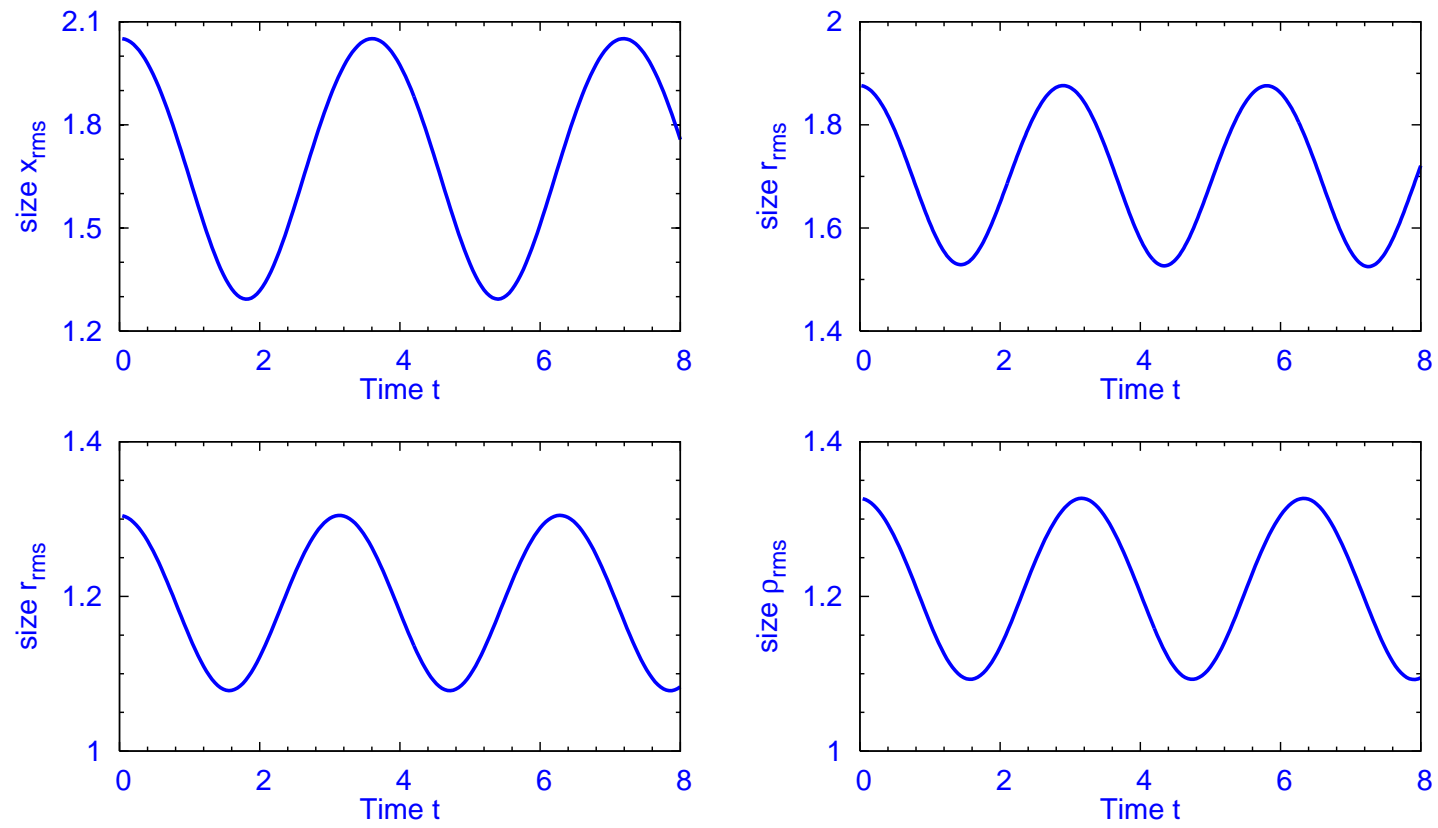

Fig. 5. (Color online) Plot of rms size vs. time for non-stationary oscillation of the system obtained by running the real-time programs for (a) 1D case (using program realtime1d.F with $\aleph=62.742$ ), (b) spherically-symmetric case (using program realtimesph.F with $\aleph=125.484$ ), (c) $2 \mathrm{D}$ circularly-symmetric case (using program realtime2d.F with $\aleph=12.5484$ and $\kappa=1$ ), and (d) 3D axially-symmetric case (using program realtimeaxial.F with $\aleph=18.81$ and $\kappa=1, \lambda=4$ ). The oscillation is started during time evolution by suddenly reducing the nonlinearity $\aleph$ to half after the formation of the stationary condensate.

spatial derivative parts are treated by the Crank-Nicolson method. Different spatial derivative and nonderivative parts are dealt in independent steps. This, so called split-step, method leads to highly stable and accurate results.

We considered two types of time iterations - real-time propagation and imaginary-time propagation. In the real-time propagation time evolution is performed with the original complex equation. The numerical algorithm in this case requires the use of complex variable but produces solution of non-stationary problems. In the imaginary-time propagation, the time variable is replaced by i $(=\sqrt{-1})$ times a new time variable, consequently the GP equation becomes real. The numerical solution of this equation can no longer yield the solution of non-stationary problems; but yields very accurate solution of stationary ground state problems only, requiring much smaller CPU time.

We provide the numerical algorithm in detail in 1D, 2D, and 3D for real- and imaginary-time propagations. We consider six different harmonic oscillator trap symmetries, e.g., a 1D trap, a circularly-symmetric 2D trap, a radially-symmetric 3D trap, an anisotropic trap in 2D, an axially-symmetric 3D trap, and an anisotropic trap in 3D. Each of these cases are treated with real- and imaginary-time propagation algorithms resulting in twelve different Fortran 77 programs supplied. We use the imaginary-time propagation programs to provide results for different stationary properties of the condensate (chemical potential, rms size, etc) in $1 \mathrm{D}, 2 \mathrm{D}$, $3 \mathrm{D}$, for different nonlinearities $\aleph$ and compare with previously obtained results [4,5]. In addition we study a non-stationary oscillation initiated by suddenly altering the nonlinearity to half its initial value on these preformed condensates using the real-time propagation programs. In addition six Fortran 90/95 programs are supplied in the case of two and three space variables.

Although the present programs are valid for the standard GP equation with cubic nonlinearity in a harmonic potential, they can be easily adopted for other types of bosonic [55] or fermionic equations [53,54] with different nonlinearities and under different types of potentials. To change the potential one should change the variable $\mathrm{V}$ in the subroutine INITIALIZE and the change in the nonlinearity can be performed in the subroutine NONLIN. 


\section{Acknowledgements}

We thank Dr. A. Gammal for helpful comments regarding the solution of the GP equation in the circularlysymmetric and axially-symmetric cases. We thank Prof. W. Bao for the hospitality at the National University of Singapore when this project was started. The research was partially supported by the CNPq and FAPESP of Brazil, and the Institute for Mathematical Sciences of the National University of Singapore. PM thanks the Third World Academy of Sciences (TWAS-UNESCO Associateship at the Center of Excellence in the South), and Department of Science and Technology, Government of India for partial support.

\section{References}

[1] F. Dalfovo, S. Giorgini, L. P. Pitaevskii, S. Stringari, Theory of Bose-Einstein condensation in trapped gases, Rev. Mod. Phys. 71 (1999) 463-512;

A. J. Leggett, Bose-Einstein condensation in the alkali gases: Some fundamental concepts, Rev. Mod. Phys. 73 (2001) 307-356.

$[2]$ L. Pitaevskii, S. Stringari, Bose-Einstein Condensation, Clarendon Press, Oxford and New York, 2003;

C. J. Pethick, H. Smith, Bose-Einstein Condensation in Dilute Gases, Cambridge University Press, Cambridge, 2002.

[3] R. P. Tiwari, A. Shukla, A basis-set based Fortran program to solve the Gross-Pitaevskii equation for dilute Bose gases in harmonic and anharmonic traps, Comp. Phys. Commun. 174 (2006) 966-982.

[4] W. Bao, W. Tang, Ground-state solution of Bose-Einstein condensate by directly minimizing the energy functional, J. Comput. Phys. 187 (2003) 230-254.

[5] B. I. Schneider, D. L. Feder, Numerical approach to the ground and excited state of a Bose-Einstein condensed gas confined in a completely anisotropic trap, Phys. Rev. A 59 (1999) 2232-2242.

[6] M. L. Chiofalo, S. Succi, M. P. Tosi, Ground state of trapped interacting Bose-Einstein condensates by an explicit imaginarytime algorithm, Phys. Rev. E 62 (2000) 7438-7444.

[7] M. M. Cerimele, M. L. Chiofalo, F. Pistella, S. Succi, M. P. Tosi, Numerical solution of the Gross-Pitaevskii equation using an explicit finite-difference scheme: An application to trapped Bose-Einstein condensates, Phys. Rev. E 62 (2000) $1382-1389$.

[8] S. L. Chang, C. S. Chien, B. W. Jeng, Computing wave functions of nonlinear Schrödinger equations: A time-independent approach, J. Comput. Phys. 226 (2007) 104-130.

[9] S. Palpacelli, S. Succi, R. Spigler, Ground-state computation of Bose-Einstein condensates by an imaginary-time quantum lattice Boltzmann scheme, Phys. Rev. E 76 (2007) 036712.

[10] M. Javidi, A. Golbabai, Numerical studies on nonlinear Schrödinger equations by spectral collocation method with preconditioning, J. Math. Analysis and Applications 333 (2007) 1119-1127.

[11] H. Q. Wang, A time-splitting spectral method for coupled Gross-Pitaevskii equations with applications to rotating BoseEinstein condensates, J. Comput and Applied Maths. 205 (2007) 88-104.

[12] M. Brtka, A. Gammal, L. Tomio, Relaxation algorithm to hyperbolic states in Gross-Pitaevskii equation, Phys. Lett. A 359 (2006) 339-344.

[13] W. Hua, X. H. Liu, P. H. Ding, Numerical solution for the Gross-Pitaevskii equation, J. Math. Chem. 40 (2006) $243-255$.

[14] Z. L. Xu, H. Han, Absorbing boundary conditions for nonlinear Schrödinger equations, Phys. Rev. E 74 (2006) 037704.

[15] J. Javanainen, J. Ruostekoski, Symbolic calculation in development of algorithms: split-step methods for the GrossPitaevskii equation, J. Phys. A 39 (2006) L179-L184.

[16] B. I. Schneider, L. A. Collins, S. X. Hu, Parallel solver for the time-dependent linear and nonlinear Schrödinger equation, Phys. Rev. E 73 (2006) 036708.

[17] S. Succi, F. Toschi, M. P. Tosi, et al., Bose-Einstein condensates and the numerical solution of the Gross-Pitaevskii equation, Computing Sci. Engineering 7 (2005) 48-57.

[18] S. M. Chang, Y. C. Kuo, W. W. Lin, et al. A continuation BSOR-Lanczos-Galerkin method for positive bound states of a multi-component Bose-Einstein condensate, J. Comput. Phys. 210 (2005) 439-458.

[19] W. Z. Bao, J. Shen, Fourth-order time-splitting Laguerre-Hermite pseudospectral method for Bose-Einstein condensates, Siam J. Sci. Computing 26 (2005) 2010-2028. 
[20] S. M. Chang, W. W. Lin, S. F. Shieh, Gauss-Seidel-type methods for energy states of a multi-component Bose-Einstein condensate, J. Comput. Phys. 202 (2005) 367-390.

[21] W. Z. Bao, Q. Du, Computing the ground state solution of Bose-Einstein condensates by a normalized gradient flow, Siam J. Sci. Computing 25 (2004) 1674-1697.

[22] M. C. Lai, C. Y. Huang, T. S. Lin, A simple Dufort-Frankel-type scheme for the Gross-Pitaevskii equation of Bose-Einstein condensates on different geometries, Numerical Methods Partial Diff. Eqs. 20 (2004) 624-638.

[23] W. Z. Bao, Ground states and dynamics of multicomponent Bose-Einstein condensates, Multiscale Modeling Simulation 2 (2004) 210-236.

[24] A. Zhou, An analysis of finite-dimensional approximations for the ground state solution of Bose-Einstein condensates, Nonlinearity 17 (2004) 541-550.

[25] Y. S. Choi, J. Javanainen, I. Koltracht, et al., A fast algorithm for the solution of the time-independent Gross-Pitaevskii equation, J. Comput. Phys. 190 (2003) 1-21.

[26] P. Muruganandam, S. K. Adhikari, Bose-Einstein condensation dynamics in three dimensions by the pseudospectral and finite-difference methods, J. Phys. B 36 (2003) 2501-2513.

[27] W. Z. Bao, S. Jin, P. A. Markowich, On time-splitting spectral approximations for the Schrödinger equation in the semiclassical regime, J. Comput. Phys. 175 (2002) 487-524.

[28] W. Z. Bao, D. Jaksch, P. A. Markowich, Numerical solution of the Gross-Pitaevskii equation for Bose-Einstein condensation, J. Comput. Phys. 187 (2003) 318-342.

[29] P. Vignolo, M. L. Chiofalo, M. P. Tosi, et al., Explicit finite-difference and particle method for the dynamics of mixed Bose-condensate and cold-atom clouds, J. Comput. Phys. 182 (2002) 368-391.

[30] S. K. Adhikari, P. Muruganandam, Bose-Einstein condensation dynamics from the numerical solution of the GrossPitaevskii equation, J. Phys. B 35 (2002) 2831-2843.

[31] M. M. Cerimele, M. L. Chiofalo, F. Pistella, Numerical solution of the stationary Gross-Pitaevskii equation: tests of a combined imaginary-time-marching technique with splitting, Nonlinear Analysis-Theory Methods Applications 47 (2001) 3345-3356.

[32] W. Z. Bao, S. Jin, P. A. Markowich, Numerical study of time-splitting spectral discretizations of nonlinear Schrödinger equations in the semiclassical regimes, Siam J. Sci. Computing 25 (2003) 27-64.

[33] S. K. Adhikari, Numerical solution of the two-dimensional Gross-Pitaevskii equation for trapped interacting atoms, Phys. Lett. A 265 (2000) 91-96.

[34] A. Gammal, T. Frederico, L. Tomio, Improved numerical approach for the time-independent Gross-Pitaevskii nonlinear Schrödinger equation, Phys. Rev. E 60 (1999) 2421-2424.

[35] A. L. Fetter, Variational study of dilute bose condensate in a harmonic trap, J. Low Temp. Phys. 106 (1997) $643-652$.

[36] R. J. Dodd, Approximate solutions of the nonlinear Schrödinger equation for ground and excited states of Bose-Einstein condensates, J. Res. National Inst. Standards Tech. 101 (1996) 545-552.

[37] S. K. Adhikari, Numerical study of the spherically symmetric Gross-Pitaevskii equation in two space dimensions, Phys. Rev. E 62 (2000) 2937-2944.

[38] S. K. Adhikari, Numerical study of the coupled time-dependent Gross-Pitaevskii equation: Application to Bose-Einstein condensation, Phys. Rev. E 63 (2001) 056704.

[39] F. Dalfovo, S. Stringari, Bosons in anisotropic traps: Ground state and vortices, Phys. Rev. A 53 (1996) $2477-2485$.

[40] Q. X. Yuan, G. H. Ding, Computing ground state solution of Bose-Einstein condensates trapped in one-dimensional harmonic potential, Communications Theor. Phys. 46 (2006) 873-878.

[41] M. J. Holland, J. Cooper, Expansion of a Bose-Einstein condensate in a harmonic potential, Phys. Rev. A 53 (1996) R1954-R1957.

[42] M. M. Cerimele, F. Pistella, S. Succi, Particle-inspired scheme for the Gross-Pitaevski equation: An application to BoseEinstein condensation, Comput. Phys. Commun. 129 (2000) 82-90.

[43] S. Palpacelli, S. Succi, Quantum lattice Boltzmann simulation of expanding Bose-Einstein condensates in random potentials, Phys. Rev. E 77 (2008) 066708.

[44] A. Aftalion, Q. Du, Vortices in a rotating Bose-Einstein condensate: Critical angular velocities and energy diagrams in the Thomas-Fermi regime, Phys. Rev. A 64 (2001) 063603.

[45] L. Lehtovaara, J. Toivanen, J. Eloranta, Solution of time-independent Schrödinger equation by the imaginary time propagation method, J. Comput. Phys. 221 (2007) 148-157. 
[46] M. Edwards, K. Burnett, Numerical solution of the nonlinear Schrödinger equation for small samples of trapped neutral atoms, Phys. Rev. A 51 (1995) 1382-1386.

[47] P. A. Ruprecht, M. J. Holland, K. Burnett, M. Edwards, Time-dependent solution of the nonlinear Schrödinger equation for Bose-condensed trapped neutral atoms, Phys. Rev. A 51 (1995) 4704-4711.

[48] R. Baer, Accurate and efficient evolution of nonlinear Schrödinger equations, Phys. Rev. A 62 (2000) 063810.

[49] S. E. Koonin, D. C. Meredith, Computational Physics: Fortran version, Addison-Wesley, Reading, 1990.

[50] W. F. Ames, Numerical Methods for Partial Differential Equations, 3rd Ed, Academic Press, New York, 1992.

[51] R. Dautray, J. L. Lions, Mathematical Analysis and Numerical Methods for Science and Technology, vol 6, Springer Verlag, Berlin, 1993, Chapter XX, Sec. 2.

[52] S. K. Adhikari, Mean-field description of collapsing and exploding Bose-Einstein condensates, Phys. Rev. A 66 (2002) 013611;

S. K. Adhikari, Dynamics of a collapsing and exploding Bose-Einstein condensed vortex state Phys. Rev. A 66 (2002) 043601.

[53] S. K. Adhikari, Nonlinear Schrödinger equation for a superfluid Fermi gas in the BCS-BEC crossover, Phys. Rev. A 77 (2008) 045602.

[54] S. K. Adhikari, Superfluid Fermi-Fermi mixture: Phase diagram, stability, and soliton formation, Phys. Rev. A 76 (2007) 053609 .

[55] L. Salasnich, S. K. Adhikari, F. Toigo, Self-bound droplet of Bose and Fermi atoms in one dimension: Collective properties in mean-field and Tonks-Girardeau regimes, Phys. Rev. A 75 (2007) 023616;

M. Girardeau, Relationship between systems of impenetrable bosons and fermions in one dimension, J. Math. Phys. 1 (1960) 516-523

M. D. Girardeau, Permutation symmetry of many-particle wave functions, Phys. Rev. 139 (1965) B500-B508.

[56] S. K. Adhikari, Fermionic bright soliton in a boson-fermion mixture, Phys. Rev. A 72 (2005) 053608;

S. K. Adhikari, Stabilization of bright solitons and vortex solitons in a trapless three-dimensional Bose-Einstein condensate by temporal modulation of the scattering length, Phys. Rev. A 69 (2004) 063613;

S. K. Adhikari, Mean-field model of interaction between bright vortex solitons in Bose-Einstein condensates, New J. Phys. 5 (2003) 137;

S. Rajendran, P. Muruganandam, M. Lakshmanan, Non-stationary excitations in Bose-Einstein condensates under the action of periodically varying scattering length with time-dependent frequencies, Physica D 227 (2007) 1-7.

[57] S. K. Adhikari, Free expansion of attractive and repulsive Bose-Einstein condensed vortex states, Phys. Rev. A 65 (2002) 033616.

[58] M. Kozuma, L. Deng, E. W. Hagley, J. Wen, R. Lutwak, K. Helmerson, S. L. Rolston, W. D. Phillips, Coherent Splitting of Bose-Einstein Condensed Atoms with Optically Induced Bragg Diffraction, Phys. Rev. Lett. 82 (1999) 871-880.

[59] L. V. Hau, B. D. Busch, C. Liu, Z. Dutton, M. M. Burns, J. A. Golovchenko, Near-resonant spatial images of confined Bose-Einstein condensates in a 4-Dee magnetic bottle, Phys. Rev. A 58 (1998) R54-R57. 